\title{
Neutron-capture elements across the Galactic thin disk using Cepheids ${ }^{\star}$
}

\author{
R. da Silva ${ }^{1,2,3}$, B. Lemasle ${ }^{4}$, G. Bono ${ }^{2,3}$, K. Genovali ${ }^{2}$, A. McWilliam ${ }^{5}$, S. Cristallo ${ }^{6,7}$, M. Bergemann ${ }^{8}$, \\ R. Buonanno ${ }^{2,6}$, M. Fabrizio ${ }^{1,6}$, I. Ferraro ${ }^{3}$, P. François ${ }^{9,10}$, G. Iannicola ${ }^{3}$, L. Inno ${ }^{8}$, C. D. Laney ${ }^{11,12}$, \\ R.-P. Kudritzki ${ }^{13,14,15}$, N. Matsunaga ${ }^{16}$, M. Nonino ${ }^{17}$, F. Primas ${ }^{18}$, N. Przybilla ${ }^{19}$, M. Romaniello ${ }^{18,20}$, \\ F. Thévenin ${ }^{21}$, and M. A. Urbaneja ${ }^{19}$
}

1 ASI Science Data Center, via del Politecnico snc, 00133 Rome, Italy e-mail: ron.oliveirasilva@gmail.com

2 Dipartimento di Fisica, Università di Roma Tor Vergata, via della Ricerca Scientifica 1, 00133 Rome, Italy

3 INAF-Osservatorio Astronomico di Roma, via Frascati 33, 00078 Monte Porzio Catone, Rome, Italy

4 Anton Pannekoek Institute for Astronomy, University of Amsterdam, Science Park 904, PO Box 94249, 1090 GE, Amsterdam, The Netherlands

5 The Observatories of the Carnegie Institute of Washington, 813 Santa Barbara Street, Pasadena, CA 91101, USA

${ }^{6}$ INAF-Osservatorio Astronomico di Teramo, via Mentore Maggini s.n.c., 64100 Teramo, Italy

7 INFN Sezione Napoli, Napoli, Italy

8 Max-Planck-Institut für Astronomy, 69117 Heidelberg, Germany

9 GEPI, Observatoire de Paris, CNRS, Université Paris Diderot, Place Jules Janssen, 92190 Meudon, France

10 UPJV, Université de Picardie Jules Verne, 33 rue St. Leu, 80080 Amiens, France

11 Department of Physics and Astronomy, N283 ESC, Brigham Young University, Provo, UT 84601, USA

12 South African Astronomical Observatory, PO Box 9, Observatory 7935, South Africa

13 Institute for Astronomy, University of Hawaii, 2680 Woodlawn Drive, Honolulu, HI 96822, USA

14 Max-Planck-Institute for Astrophysics, Karl-Schwarzschild-Str.1, 85741 Garching, Germany

15 University Observatory Munich, Scheinerstr. 1, 81679 Munich, Germany

16 Department of Astronomy, School of Science, The University of Tokyo, 7-3-1 Hongo, Bunkyo-ku, Tokyo 113-0033, Japan

17 INAF-Osservatorio Astronomico di Trieste, via G. B. Tiepolo 11, 34143 Trieste, Italy

18 European Southern Observatory, Karl-Schwarzschild-Str. 2, 85748 Garching bei München, Germany

19 Institute for Astro- and Particle Physics, University of Innsbruck, Technikerstr. 25/8, 6020 Innsbruck, Austria

${ }^{20}$ Excellence Cluster Universe, Boltzmannstr. 2, 85748 Garching bei München, Germany

${ }^{21}$ Laboratoire Lagrange, CNRS/UMR 7293, Observatoire de la Côte d'Azur, Bd de l'Observatoire, CS 34229, 06304 Nice, France

Received 3 September 2015 / Accepted 19 October 2015

\section{ABSTRACT}

We present new accurate abundances for five neutron-capture elements ( $\mathrm{Y}, \mathrm{La}, \mathrm{Ce}, \mathrm{Nd}, \mathrm{Eu})$ in 73 classical Cepheids located across the Galactic thin disk. Individual abundances are based on high spectral resolution $(R \sim 38000)$ and high signal-to-noise ratio $(S / N \sim$ 50-300) spectra collected with UVES at ESO VLT for the DIONYSOS project. Taking into account similar Cepheid abundances provided by our group (111 stars) and available in the literature, we end up with a sample of 435 Cepheids covering a broad range in iron abundances $(-1.6<[\mathrm{Fe} / \mathrm{H}]<0.6)$. We found, via homogeneous individual distances and abundance scales, well-defined gradients for the above elements. However, the slope of the light $s$-process element (Y) is at least a factor of two steeper than the slopes of heavy $s$ - $(\mathrm{La}, \mathrm{Ce}, \mathrm{Nd})$ and $r$ - $(\mathrm{Eu})$ process elements. The $s$-to- $r$ abundance ratio $([\mathrm{La} / \mathrm{Eu}])$ of Cepheids shows a welldefined anticorrelation with both $\mathrm{Eu}$ and $\mathrm{Fe}$. On the other hand, Galactic field stars attain an almost constant value and display a mild enhancement in La only when they approach solar iron abundance. The [Y/Eu] ratio shows slight evidence of a correlation with $\mathrm{Eu}$ and, in particular, with iron abundance for field Galactic stars. We also investigated the s-process index ([hs/ls]) and we found a well-defined anticorrelation, as expected, between $[\mathrm{La} / \mathrm{Y}]$ and iron abundance. Moreover, we found a strong correlation between $[\mathrm{La} / \mathrm{Y}]$ and $[\mathrm{La} / \mathrm{Fe}]$ and, in particular, a clear separation between Galactic and Sagittarius red giants. Finally, the comparison between predictions for low-mass asymptotic giant branch stars and the observed $[\mathrm{La} / \mathrm{Y}]$ ratio indicate a very good agreement over the entire metallicity range covered by Cepheids. However, the observed spread at fixed iron content is larger than predicted by current models.

Key words. stars: abundances - stars: variables: Cepheids - stars: oscillations - open clusters and associations: general Galaxy: disk

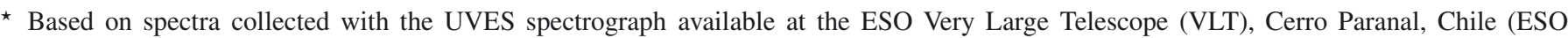
Proposals: 081.D-0928(A), PI: S. Pedicelli; 082.D-0901(A), PI: S. Pedicelli; 089.D-0767(C), PI: K. Genovali).

$\star \star$ Tables 2, 3, 4, and 7 are only available at the CDS via anonymous ftp to cdsarc.u-strasbg.fr (130.79.128.5) or via

http://cdsarc.u-strasbg.fr/viz-bin/qcat?J/A+A/586/A125
} 


\section{Introduction}

The use of classical Cepheids as solid distance indicators dates back to more than one century ago (Leavitt 1908; Leavitt \& Pickering 1912). The evidence that individual distances could be estimated on the basis of the pulsation period and the mean magnitude has also made Cepheids very popular stellar tracers. However, the use of classical Cepheids as tracers of young stellar populations is more recent and dates back to the seminal investigations by Kraft \& Schmidt (1963). To constrain the geometry, the rotation, and the density distribution of the Galactic thin disk they used distances and radial velocities of a sample of 267 Cepheids. The above empirical evidence was soundly supplemented by pioneering evolutionary and pulsation investigations suggesting that the pulsation period of classical Cepheids is highly anticorrelated with their age (Kippenhahn et al. 1969; Meyer-Hofmeister 1969).

During the last twenty years, classical Cepheids have been the crossroad of several photometric and spectroscopic investigations. Thanks to the new photometric surveys in the optical - OGLE-IV (Udalski et al. 2015) - and near-infrared (NIR) IRIS (Miville-Deschênes \& Lagache 2005) and VVV (Minniti et al. 2010) - classical Cepheids have been identified and characterized in the thin disk and in the different components of the Galactic spheroid hosting young-stellar populations: i) inner disk (Matsunaga et al. 2013; Inno et al., in prep.); ii) nuclear bulge (Matsunaga et al. 2011); iii) beyond the nuclear bulge (Feast et al. 2014; Dékány et al. 2015); and iv) outer disk (e.g., Metzger et al. 1992; Pont et al. 2001). This suggests that they can provide solid constraints on the impact that environment has on the recent star formation episodes of the Galactic thin disk.

The use of high-resolution spectrographs revealed that classical Cepheids are also excellent tracers of the chemical enrichment of intermediate-mass stars across the thin disk (Kraft 1965; Conti \& Wallerstein 1969). In spite of these indisputable advantages in using classical Cepheids as stellar tracers, the first detailed investigation of the thin disk iron gradient dates back to Harris (1981) and Harris \& Pilachowski (1984). More recent theoretical and empirical investigations revealed that classical Cepheids, i.e., yellow and red giants (RGs) and supergiants, display hundreds of iron lines in their spectra (Andrievsky et al. 2002a,b,c, 2004; Yong et al. 2006; Luck et al. 2006, 2011; Lemasle et al. 2007, 2008; Pedicelli et al. 2010; Luck \& Lambert 2011; Genovali et al. 2013, 2014), dozens of $\alpha$-element (e.g., Andrievsky et al. 2004; Luck \& Lambert 2011; Lemasle et al. 2013; Genovali et al. 2015), and iron peak lines (e.g., Andrievsky et al. 2004; Luck \& Lambert 2011), together with a well-defined continuum. They are also excellent laboratories to constrain the impact of non-LTE effects in the abundance of both CNO (Luck et al. 2011; Martin et al. 2015) and Na (Takeda et al. 2013; Genovali et al. 2015) in RGs. Cepheids have also been identified as able to constrain the abundance of lithium in young stellar populations (Kovtyukh et al. 2005; Luck \& Lambert 2011).

The above investigations move along a well-defined path concerning the elemental abundance analysis and the chemical enrichment history of the thin disk. In this context neutron capture elements play a crucial role since they trace the yields of a broad range of stellar structures (see, e.g., Sneden et al. 2008). They are typically split into two different groups, the heavy elements formed either via slow neutron-capture (" $s$-process") or rapid neutron capture (" $r$-process"), i.e., either slow or rapid compared to the $\beta$-decay time scale.

The "main $s$-process" is considered to occur in asymptotic giant branch (AGB) stars during recurrent thermal pulses
(Gallino et al. 1998; Busso et al. 1999). On the other hand, the "weak $s$-process" takes place in massive fast evolving primitive stars and produces elements with atomic mass number smaller than $A=90$ (Raiteri et al. 1993; Pignatari et al. 2010). The astrophysical sites of the $r$-process elements are even more complex. Indeed, the recent literature on the nucleosynthesis of $r$ elements is quite rich. It has been suggested that they can be produced by core-collapse supernovae ( $\mathrm{SNe}$ ) with a mass on the order of $20 M_{\odot}$ (Thielemann et al. 2011; Wanajo 2013; Tsujimoto \& Nishimura 2015; Nishimura et al. 2015); by core-collapse SNe of very massive stars $\left(25 \leq M / M_{\odot} \leq 45\right.$, Boyd et al. 2012), by electron capture $\mathrm{SNe}$ in intermediate-mass stars $\left(8-10 M_{\odot}\right.$, Woosley \& Heger 2015), and by more complex astrophysical mechanisms such as the neutrino driven wind leading to the merging of neutron stars (e.g., Wanajo \& Janka 2012; Berger et al. 2013).

The abundance of s- and $r$-process elements in Galactic classical Cepheids has already been discussed in several recent papers (Andrievsky et al. 2004; Luck et al. 2011; Luck \& Lambert 2011; Lemasle et al. 2013). However, we still lack a homogeneous and detailed analysis of weak and main $s$-process elements in the Galaxy and their dependence on the iron abundance - a detailed analysis of $s$ and $r$ elements of RGs in the Sagittarius dwarf galaxy has been performed by McWilliam et al. (2013, hereafter M13). Moreover, and even more importantly, we still lack a quantitative analysis of the spatial abundance pattern of $s$ - and $r$-process elements and, in particular, the possible occurrence of an age dependence.

In this investigation we focus our attention on the abundance of one light (Y) and three heavy ( $\mathrm{La}, \mathrm{Ce}, \mathrm{Nd}$ ) dominated $s$-process elements, and a single $r$-process dominated element $(\mathrm{Eu})$. The abundances of 73 Galactic Cepheids of our sample were complemented with the abundances of 362 Cepheids available in the literature.

The structure of the paper is the following. In Sect. 2 we discuss the spectra we collected and the approach we adopted for data reduction and analysis. In this section we also mention the different samples of Cepheid abundances based on highresolution spectra and available in the literature, and the approach we adopted to provide a homogeneous metallicity scale. In Sect. 3 we discuss the radial gradients of [element/H] ratios for neutron-capture elements and their comparison with iron and $\alpha$-element gradients. In this section we also discuss the radial gradients of [element/Fe] ratios and their age dependence. In Sect. 4 we present s- and $r$-process element abundances and compare their distribution with dwarf and giant stars available in the literature. The differentiation between weak and main $s$-process elements and the comparison with the literature are also discussed in this section. The summary of the results of the current investigation are given in Sect. 5 together with a brief outline of the future development of this project.

\section{Observations, data reduction, and analysis}

\subsection{Spectroscopic data}

In this work we used the same high-resolution $(R \sim 38000)$ and high signal-to-noise ratio $(\mathrm{S} / \mathrm{N})$ spectra reported in Genovali et al. (2014, hereafter G14) in our determination of iron abundances and atmospheric parameters, and in Genovali et al. (2015, hereafter G15) in our study of $\alpha$-element abundances. A total of 122 spectra of 75 Galactic Cepheids were collected with the UVES spectrograph at the ESO VLT (Cerro Paranal, Chile) using two different instrument settings: i) with the first we 
R. da Silva et al.: Neutron-capture elements across the Galactic thin disk using Cepheids
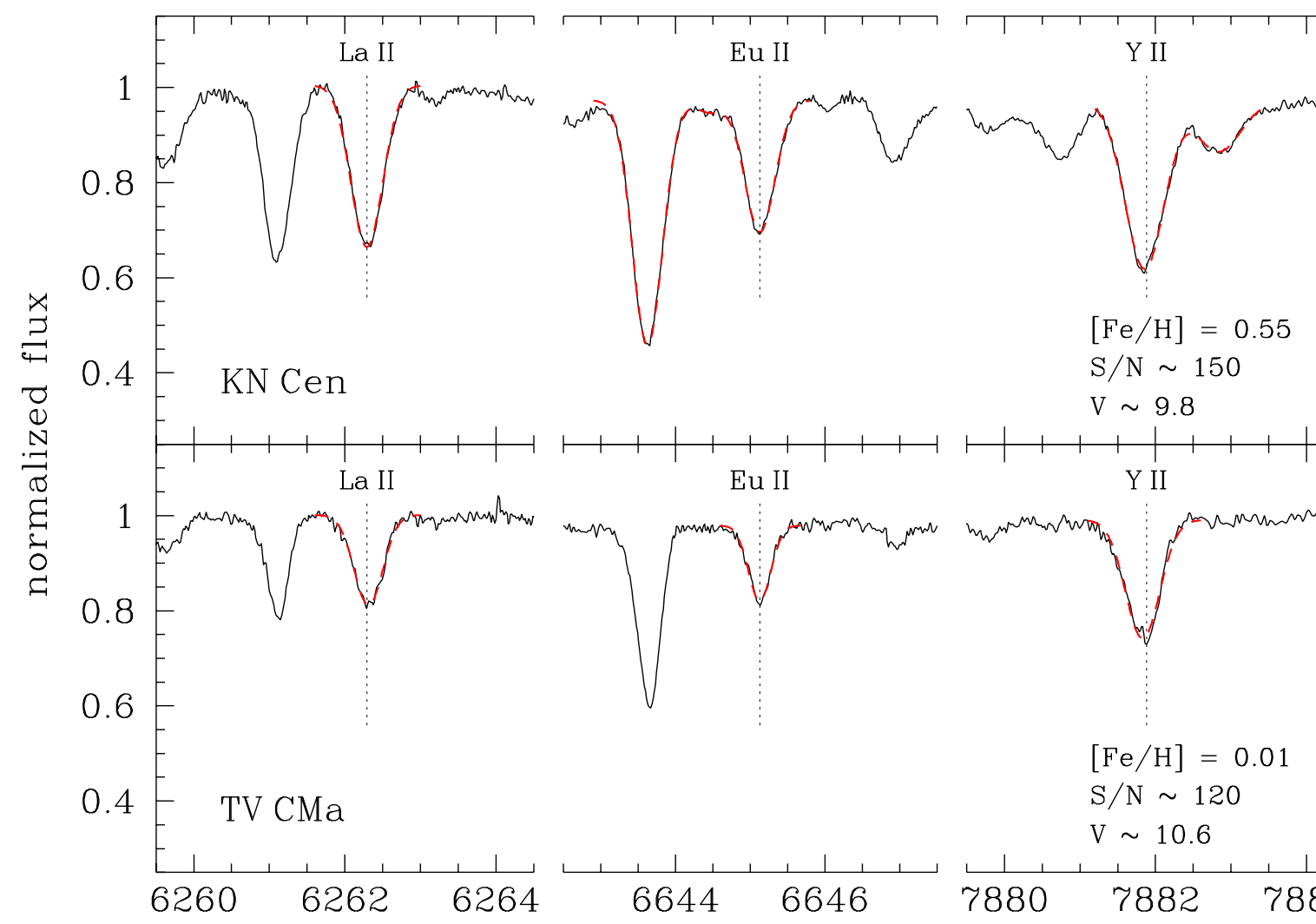

$\mathrm{Fe} / \mathrm{H}]=0.55$

$\mathrm{S} / \mathrm{N} \sim 150$
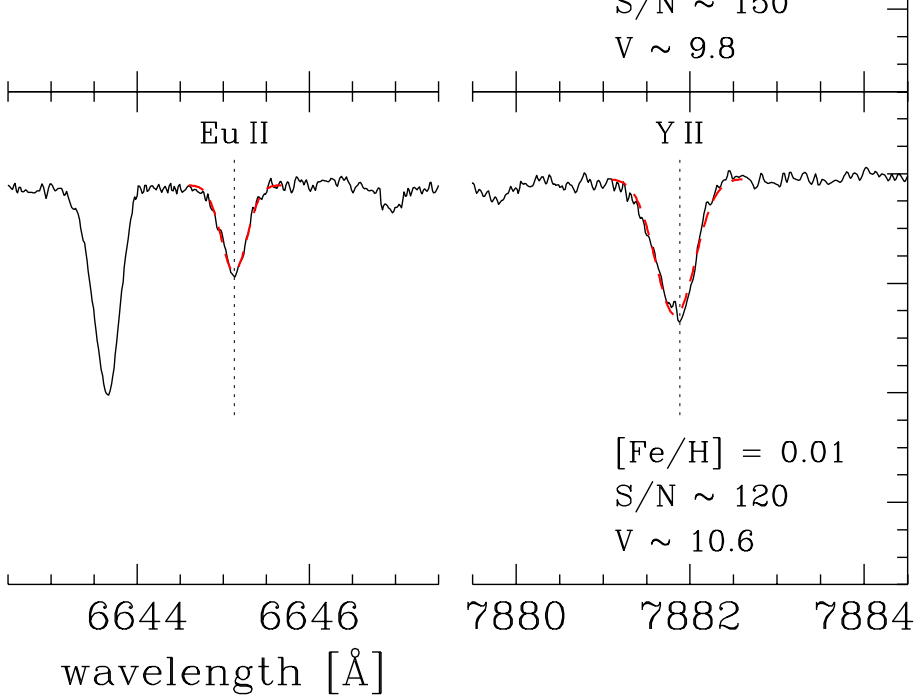

Fig. 1. High-resolution $(R \sim 38000)$ UVES spectrum of KN Cen and TV CMa. The apparent visual magnitude and the $\mathrm{S} / \mathrm{N}$ in the spectral range $\lambda \sim 5650-7500 \AA$ are also labeled. The vertical dashed lines show some of the spectral lines (La II 6262.29, Eu II 6645.13, Y II 7881.88 $\AA$ ) adopted to estimate the abundances.

collected 80 spectra of 74 stars in the wavelength ranges of

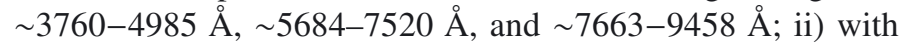
the second we collected 42 spectra of a control sample of 11 Cepheids in the wavelength ranges of $\sim 4786-5750 \AA$ and 5833-6806 $\AA$. For more details on the instrument settings used we refer the reader to the papers G14 and G15.

In the same way as was done in the papers mentioned above, we used the 11 Cepheids (V340 Ara, AV Sgr, VY Sgr, UZ Sct, Z Sct, V367 Sct, WZ Sgr, XX Sgr, KQ Sco, RY Sco, and V500 Sco) as a control sample. For these stars we have from four to six spectra each, collected with both the instrumental configurations (with the exception of V500 Sco, which has four spectra collected only with the second instrument setting). For the same reasons reported in G15, the stars BB Gem and GQ Ori (both observed using the first instrument setting) were not included in the current analysis, thus we are left with 73 stars. The $\mathrm{S} / \mathrm{N}$ values are typically better than $\sim 100$ per extracted pixel for all the échelle orders in the case of the first instrumental configuration (see examples in Fig. 1) and range from $\sim 50$ to roughly 300 for the second. All the spectra were reduced using the ESO UVES pipeline Reflex v2.1 (Ballester et al. 2011).

\subsection{Atmospheric parameters and abundances}

We adopted the same iron abundances and atmospheric parameters derived by G14. The iron abundances are based on the equivalent width (EW) of about 100-200 Fe I and about 20-40 Fe II lines, the number of lines depending on the spectral range used. The number of lines also varies according to the metallicity and to the spectral type of the star (at the time of the observation). To determine the atmospheric parameters, we set a limit of $E W<120 \mathrm{~m} \AA$ to remain in the linear part of the curve of growth. For the objects where the number of weak lines was too small, we increased the limit to $180 \mathrm{~m} \AA$. This slightly increases the uncertainties affecting the correlated atmospheric parameters, namely the effective temperature $\left(T_{\text {eff }}\right)$ and the microturbulent velocity $\left(v_{t}\right)$. For more details on the impact that typical uncertainties on $T_{\text {eff }}$, surface gravity $(\log g)$, and $v_{t}$ have on the iron abundances, see Table 2 in G14.

The $T_{\text {eff }}$ of individual spectra was estimated using the line depth ratio (LDR) method and calibrations derived by Kovtyukh $\&$ Gorlova (2000). We adopted these calibrations because those provided by Kovtyukh (2007) are not publicly available; the difference in the temperature scale between the two calibrations is quite modest (see Fig. 4 in Kovtyukh 2007). These calibrations have been criticized by Lyubimkov et al. (2010) and by Luck (2014), who suggest that the effective temperatures higher than 6500-6800 K have been overestimated. In our sample only a minor fraction (6 out of 73 Cepheids) has an effective temperature higher than $6300 \mathrm{~K}$. We note that the estimated values of $T_{\text {eff }}$ were validated by verifying that the Fe I abundances do not depend on the excitation potential $\left(\chi_{\mathrm{ex}}\right)$, i.e., the slope of $[\mathrm{Fe} \mathrm{I} / \mathrm{H}]$ vs. $\chi_{\mathrm{ex}}$ should be close to zero.

The $\log g$ was derived through the ionization equilibrium between $\mathrm{Fe} \mathrm{I}$ and $\mathrm{Fe}$ II lines, and $v_{t}$ was derived by minimizing the slope in the $[\mathrm{Fe} \mathrm{I}]$ vs. EW plot. This means that the $\log g$ value is changed until the Fe I and Fe II lines provide the same abundance, while the $v_{t}$ value is changed until the dependence of the 
derived abundances on the EWs is removed. Indeed, weak and strong lines are supposed to provide the same elemental abundances. In this context it is worth mentioning that we are dealing with radial variables and the quoted physical parameters $\left(T_{\text {eff }}\right.$, $\left.\log g, v_{t}\right)$ undergo cyclic variation along the pulsation cycle. The internal consistency of the adopted values was validated by G14 using the control sample of Cepheids, i.e., the objects for which we have from four to six spectra.

For the abundance of the neutron-capture elements (Y, La, $\mathrm{Ce}, \mathrm{Nd}$, and $\mathrm{Eu}$ ), we used the linelist provided by Lemasle et al. (2013) with the same atomic parameters $\left(\chi_{\mathrm{ex}}\right.$ and $\left.\log g f\right)$ listed in their Table A.1, but with small differences in the number of lines. We used six Y II lines (5119.12, 5289.81, 5402.77, 5509.91, 5728.89, and $7881.88 \AA$ ) instead of seven (the line at $6795.41 \AA$ was not used because the abundances derived using this line are systematically smaller than those derived using the other six lines). We also used the six lines for La II $(5114.56,5290.82$, 5805.77, 6262.29, 6390.48, and 6774.27 $\AA$ ), three lines for Ce II (4562.37, 5518.49, and $6043.39 \AA$ ) instead of four (the abundance provided by the line at $4486.91 \AA$ very often disagreed with the values provided by the other lines, and so was not used), the six Nd II lines (4959.12, 5092.79, 5130.59, 5181.17, 5431.52, and 6740.08 $\AA$ ), and the two for Eu II (6437.64 and $6645.13 \AA$ ).

As previously done in G15 for the $\alpha$-elements, the EWs for these neutron-capture elements were measured using the Automatic Routine for line Equivalent widths in stellar Spectra (ARES, Sousa et al. 2007), and double-checked using the splot task of IRAF $^{1}$. Again, the internal dispersion is smaller than $6 \mathrm{~m} \AA$ and there is no evidence of systematics.

The abundances were derived with the calrai spectrum synthesis package, originally developed by Spite (1967) and regularly improved since then. The package allows us to compute synthetic spectra by interpolating over a large grid of hydrostatic, LTE, and plane-parallel or spherical stellar atmospheres models (MARCS, Gustafsson et al. 2008).

For all the elements studied here, we assumed the standard solar abundances provided by Grevesse et al. (1996), namely $A(\mathrm{Fe})_{\odot}=7.50, A(\mathrm{Y})_{\odot}=2.24, A(\mathrm{La})_{\odot}=1.17, A(\mathrm{Ce})_{\odot}=1.58$, $A(\mathrm{Nd})_{\odot}=1.50$, and $A(\mathrm{Eu})_{\odot}=0.51$. Recent spectroscopic estimates of solar abundances by Scott et al. (2015) and by Grevesse et al. (2015) indicate very similar abundances. Indeed, the difference in dex ranges from +0.01 for $\mathrm{Eu}$ to -0.03 for $\mathrm{Fe}$ and $\mathrm{Y}$, to -0.06 for $\mathrm{La}$, and to -0.08 for $\mathrm{Nd}$, while the new $\mathrm{Ce}$ abundance is identical to the old one.

\subsubsection{Hyperfine structure and isotopic splitting}

Several lines used in our abundance analysis are affected by hyperfine structure (HFS) and/or isotopic splitting in the line profile. We searched in the literature for atomic data required to compute the fine-structure components that form these lines. We found that several of them are already available and for those that are not available we adopted the same approach discussed in M13 to compute the HFS. The atomic data required to compute the HFS of La and Eu were taken from Lawler et al. (2001a,b), respectively. For the La line at $6774.27 \AA$ we adopted the HFS already computed by M13. The same outcome applies to Y: we adopted the HFS data given by M13, but they are only available for three (out of six) lines of this element $(\lambda=5119.12,5289.81$, $5728.89 \AA)$.

\footnotetext{
1 Image Reduction and Analysis Facility, distributed by the National Optical Astronomy Observatories (NOAO), USA.
}

Table 1. Mean differences in the abundances derived before and after accounting for the HFS.

\begin{tabular}{lccc}
\hline \hline Species & $\lambda[\AA]$ & {$[\mathrm{X} / \mathrm{H}]_{\mathrm{HFS}}-[\mathrm{X} / \mathrm{H}]_{\mathrm{no} \mathrm{HFS}}$} & $\begin{array}{c}\text { Correction } \\
(\min , \max )\end{array}$ \\
\hline Y II & 5119.12 & $+0.013 \pm 0.006$ & $0.00,+0.03$ \\
Y II & 5289.81 & $+0.008 \pm 0.005$ & $0.00,+0.02$ \\
Y II & 5728.89 & $+0.008 \pm 0.005$ & $0.00,+0.02$ \\
La II & 5114.56 & $-0.172 \pm 0.128$ & $-0.45,-0.01$ \\
La II & 5290.82 & $-0.010 \pm 0.013$ & $-0.04,+0.01$ \\
La II & 5805.77 & $-0.129 \pm 0.098$ & $-0.53,0.00$ \\
La II & 6262.29 & $-0.211 \pm 0.178$ & $-0.90,-0.01$ \\
La II & 6390.48 & $-0.004 \pm 0.009$ & $-0.03,+0.01$ \\
La II & 6774.27 & $+0.050 \pm 0.050$ & $-0.17,+0.11$ \\
Eu II & 6437.64 & $-0.006 \pm 0.008$ & $-0.03,+0.01$ \\
Eu II & 6645.13 & $-0.023 \pm 0.021$ & $-0.10,+0.01$ \\
\hline
\end{tabular}

Notes. The quoted errors in the Col. 3 represent the dispersion around the mean, and Col. 4 lists the minimum and maximum HFS corrections applied to the abundances.

No atomic data have been found in the literature for our lines of $\mathrm{Ce}$ and $\mathrm{Nd}$. Only the odd isotopes ${ }^{143} \mathrm{Nd}$ and ${ }^{145} \mathrm{Nd}$ have HFS, but their effects can be safely ignored - in the solar system these isotopes constitute only $20.5 \%$ of the total $\mathrm{Nd}$ abundance, their lines are very narrow, and recent laboratory transition probabilities by Den Hartog et al. (2003) indicate no evident HFS structure for more than 700 lines of Nd II.

\subsubsection{Abundances corrected from HFS}

We derived the abundances of the current sample of 73 Cepheids by accounting for the HFS of the elements and lines mentioned in the previous section. In order to quantify the effects of the HFS on the derived abundances and their dependence on other parameters, we performed a comparison between our abundance results before and after performing the HFS analysis. The mean differences in abundance are summarized in Table 1 . The differences are much larger for some of the La lines, but they are very close to zero for both Y and Eu lines. For the possible dependences on other parameters, we found that these differences and their dispersions become smaller with increasing $T_{\text {eff }}$ (specially for $T_{\text {eff }}>5500 \mathrm{~K}$ ), with increasing surface gravity (specially for $\log g>0.5)$, and with decreasing pulsation period $(\log P<1.0)$. No clear correlation with metallicity is observed.

Finally, we note that the lines selected to measure n-capture elements are typically weak (smaller than $180 \mathrm{~m} \AA$ ) and unsaturated. This means that the derived abundances for $\mathrm{La}, \mathrm{Ce}, \mathrm{Nd}$, and Eu are marginally affected by HFS substructure, while for $\mathrm{Y}$ they are small (see Table 1).

Table 2 lists the abundances from individual spectra. Column 3 shows the iron abundances derived by G14, and Col. 4 the number of Fe I and Fe II lines used. The other columns show our results for the abundances of $\mathrm{Y}, \mathrm{La}, \mathrm{Ce}, \mathrm{Nd}$, and Eu corrected for HFS when possible, together with the number of lines used. In Table 3 we list the mean abundances computed for the stars with multiple spectra. The HFS data that we adopted are listed in Table 4 for the Y, La, and Eu lines.

\subsection{Data available in the literature}

We compared our abundance estimates (corrected for HFS) with the results provided by similar studies available in the literature: Lemasle et al. (2013, hereafter LEM), Luck et al. (2011, LII), Luck \& Lambert (2011, LIII), and Yong et al. (2006, YON). 
Table 5. Abundance difference of stars in common among the current sample and other data sets.

\begin{tabular}{ccrc}
\hline \hline $\begin{array}{c}\text { Abundance } \\
\text { ratio }\end{array}$ & Data sets $^{1}$ & \multicolumn{1}{c}{$\begin{array}{c}\text { Zero-point } \\
\text { difference }\end{array}$} & $N_{\text {Common }}$ \\
\hline$[\mathrm{Fe} / \mathrm{H}]$ & LII-G14 & $-0.05 \pm 0.11$ & 45 \\
{$[\mathrm{Fe} / \mathrm{H}]$} & LIII-G14 & $0.03 \pm 0.08$ & 33 \\
{$[\mathrm{Fe} / \mathrm{H}]$} & LII-LEM & $0.08 \pm 0.12$ & 51 \\
{$[\mathrm{Fe} / \mathrm{H}]$} & LIII-YON & $0.34 \pm 0.20$ & 20 \\
{$[\mathrm{Y} / \mathrm{H}]$} & LII-TS & $0.21 \pm 0.20$ & 37 \\
{$[\mathrm{Y} / \mathrm{H}]$} & LIII-TS & $0.15 \pm 0.18$ & 34 \\
{$[\mathrm{Y} / \mathrm{H}]$} & LII-LEM & $0.09 \pm 0.15$ & 46 \\
{$[\mathrm{La} / \mathrm{H}]$} & LII-TS & $0.29 \pm 0.18$ & 40 \\
{$[\mathrm{La} / \mathrm{H}]$} & LIII-TS & $0.31 \pm 0.16$ & 34 \\
{$[\mathrm{La} / \mathrm{H}]$} & LII-LEM & $0.06 \pm 0.20$ & 47 \\
{$[\mathrm{La} / \mathrm{H}]$} & LIII-YON & $0.27 \pm 0.33$ & 16 \\
{$[\mathrm{Ce} / \mathrm{H}]$} & LII-TS & $-0.06 \pm 0.16$ & 24 \\
{$[\mathrm{Ce} / \mathrm{H}]$} & LIII-TS & $0.17 \pm 0.17$ & 19 \\
{$[\mathrm{Ce} / \mathrm{H}]$} & LII-LEM & $-0.21 \pm 0.21$ & 50 \\
{$[\mathrm{Nd} / \mathrm{H}]$} & LII-TS & $0.09 \pm 0.24$ & 42 \\
{$[\mathrm{Nd} / \mathrm{H}]$} & LIII-TS & $0.15 \pm 0.18$ & 33 \\
{$[\mathrm{Nd} / \mathrm{H}]$} & LII-LEM & $-0.08 \pm 0.29$ & 50 \\
{$[\mathrm{Eu} / \mathrm{H}]$} & LII-TS & $0.10 \pm 0.17$ & 41 \\
{$[\mathrm{Eu} / \mathrm{H}]$} & LIII-TS & $0.11 \pm 0.21$ & 34 \\
{$[\mathrm{Eu} / \mathrm{H}]$} & LII-LEM & $-0.09 \pm 0.20$ & 53 \\
{$[\mathrm{Eu} / \mathrm{H}]$} & LIII-YON & $0.12 \pm 0.25$ & 15 \\
\hline & & &
\end{tabular}

Notes. ${ }^{(1)}$ G14: Genovali et al. (2014); TS: this study; LII: Luck et al. (2011); LIII: Luck \& Lambert (2011); LEM: Lemasle et al. (2013); YON: Yong et al. (2006). For Y, La, and Eu, the differences were computed after accounting for the HFS affecting some lines of these elements in the currect sample. The quoted errors represent the dispersion around the mean.

We note that none of the quoted investigations, except for YON, takes HFS corrections into account in their analysis of Cepheid spectra.

By comparing the stars that these different data sets have in common, we evaluated the systematic differences among them. The mean differences between our measurements and those of LEM, LII, LIII, and YON range in modulus from 0.02 dex for $\mathrm{Eu}$ up to 0.31 dex for Fe. The details on these comparisons are listed in Table 5 where we show the zero-point differences obtained by G14 for the iron abundances together with our determinations for the other elements. Each pair of data sets was chosen in order to maximize the number of stars in common between them. To provide a homogeneous abundance scale for Galactic Cepheids, we applied these zero-point differences to the quoted data sets, putting them in the same scale as our current sample. The element abundances available in the literature and the rescaled values are listed in Cols. 2-15 in Table 7.

The priority in using the abundances from the literature follows the same approach adopted by G14 and by G15: first, we adopt the abundances provided by our group, i.e., this study and the results from LEM, and then those provided by the other studies, namely LIII, LII, and YON, in this order. We note that the star HQ Car was also excluded from our analysis because it was recently identified as a Type II Cepheid by Lemasle et al. (2015). The final sample has 435 Cepheids, with a homogeneous abundance scale for Fe, Y, $\mathrm{La}, \mathrm{Ce}, \mathrm{Nd}$, and $\mathrm{Eu}$.

\section{Neutron-capture element gradients}

\subsection{Neutron-capture gradients from Cepheids}

In this section we investigate the radial gradients of $\mathrm{Y}, \mathrm{La}, \mathrm{Ce}$, $\mathrm{Nd}$, and Eu across the Galactic disk using our sample of 73 classical Cepheids plus a sample of 362 Cepheids available in literature. Homogeneous iron abundances and Galactocentric distances for the entire sample were provided by G14 (see their Tables 1 and 4). The main advantage of the current approach when compared with similar investigations are the following: i) the intrinsic parameters $\left(\log g, T_{\text {eff }}, v_{t}\right)$ were estimated using the same approach; ii) elemental abundances are based on highresolution and high $\mathrm{S} / \mathrm{N}$ spectra and similar line lists; iii) individual Cepheid distances were estimated using near-infrared Period-Wesenheit relations that are reddening free and minimally affected by the metallicity (Inno et al. 2013).

In the following we discuss the radial gradients of four $s$-process (Y, La, Ce, Nd) elements and a single $r$-process element (Eu). We note that n-capture elements can be split according to solar system abundances in pure $s$-process, pure $r$-process, and mixed-parentage isotopes. Among the selected elements $\mathrm{Eu}$ is a pure $r$-process element since the $r$-fraction abundance is 97\% (Burris et al. 2000; Simmerer et al. 2004). On the other hand, the selected $s$-process elements have s-fraction abundances ranging from roughly $50 \%(\mathrm{Nd}, 58 \%$, Sneden et al. 2008) to more than $70 \%$ (Y, 72\%; La, 75\%; Ce, 81\%). The quoted $s$ - and $r$-fraction abundances should be treated cautiously since Bisterzo et al. (2011), using a different approach, found similar fractions for $\mathrm{Eu}(94 \%), \mathrm{Nd}(52 \%), \mathrm{La}(71 \%)$, and Ce (81\%), but a significantly larger s-fraction for Y $(92 \%)$.

Figure 2 shows the abundances scaled to hydrogen of Y, La, $\mathrm{Ce}, \mathrm{Nd}$, and $\mathrm{Eu}$ as a function of $R_{\mathrm{G}}$ for the final sample. Stars plotted in this figure include the current 73 Cepheids plus 38 from LEM, 263 from LIII, and 61 from LII. The abundances of the above elements are not available for the Cepheid XZCMa; therefore, we ended up with a sample of 435 stars. The individual Cepheid Galactocentric distances were estimated by G14 and assume a solar Galactocentric distance of $7.94 \pm 0.37 \pm 0.26 \mathrm{kpc}$ (Groenewegen et al. 2008; Matsunaga et al. 2013). The individual $R_{\mathrm{G}}$ values are also listed in Table 3 . The typical uncertainty on the individual distances is $\sim 5 \%$ and is mainly due to the accuracy of the zero-point in the adopted Period-Wesenheit relations (for more details see Inno et al. 2013).

The Cepheid abundances from the literature plotted in Fig. 2 were scaled adopting the zero-point differences listed in Table 5. A similar approach was adopted to scale both iron (G14) and $\alpha$-element abundances (G15). This figure also shows the linear least squares fits to the current sample of 73 Cepheids (blue solid line) and to the entire sample (435, black dashed line). To avoid thorny problems in the estimate of both the zero-point and the slope owing to possible outliers, we applied a biweight procedure (Beers et al. 1990). The slopes and the zero-points of the two radial gradients are labeled. The slopes and the zero-points of the fits based on the entire sample together with their uncertainties and standard deviations are also listed in Cols. 2-4 in Table 6.

The empirical scenario emerging from the data plotted in this figures brings to light several interesting features:

i) - Radial gradients - The five investigated neutron-capture elements display well-defined radial gradients. This evidence coupled with similar results concerning iron (see G14 and references therein), $\alpha$-elements (see G15 and references therein), and iron-group elements (LII, LIII) further 
Table 6. Slopes and zero-points of the abundance gradients as a function of the Galactocentric distance and of the pulsation period.

\begin{tabular}{|c|c|c|c|c|c|c|c|c|}
\hline $\begin{array}{l}\text { Abundance } \\
\text { ratio }\end{array}$ & Slope $^{a}$ & $\begin{array}{l}\text { Zero-point } \\
{[\text { dex }]}\end{array}$ & $\begin{array}{c}\sigma \\
{[\operatorname{dex}]}\end{array}$ & $N$ & $\begin{array}{l}\text { Slope }^{a} \\
\text { (TS) }\end{array}$ & $\begin{array}{l}\text { Slope }^{a} \\
(\text { LEM) }\end{array}$ & $\begin{array}{l}\text { Slope }^{a} \\
\text { (LII) }\end{array}$ & $\begin{array}{c}\text { Slope }^{a} \\
\text { (LIII) }\end{array}$ \\
\hline \multicolumn{9}{|c|}{ as a function of $R_{\mathrm{G}}$} \\
\hline $\begin{array}{l}{[\mathrm{Y} / \mathrm{H}]} \\
{[\mathrm{La} / \mathrm{H}]} \\
{[\mathrm{Ce} / \mathrm{H}]} \\
{[\mathrm{Nd} / \mathrm{H}]} \\
{[\mathrm{Eu} / \mathrm{H}]}\end{array}$ & $\begin{array}{l}-0.053 \pm 0.003 \\
-0.020 \pm 0.003 \\
-0.024 \pm 0.003 \\
-0.025 \pm 0.003 \\
-0.030 \pm 0.004\end{array}$ & $\begin{array}{l}0.43 \pm 0.03 \\
0.13 \pm 0.03 \\
0.20 \pm 0.03 \\
0.24 \pm 0.03 \\
0.28 \pm 0.03\end{array}$ & $\begin{array}{l}0.14 \\
0.14 \\
0.14 \\
0.13 \\
0.16\end{array}$ & $\begin{array}{l}429 \\
424 \\
421 \\
430 \\
420\end{array}$ & $\begin{array}{r}-0.033 \pm 0.007 \\
0.002 \pm 0.005 \\
0.008 \pm 0.007 \\
0.006 \pm 0.006 \\
-0.013 \pm 0.005\end{array}$ & $\begin{array}{l}-0.062 \pm 0.012 \\
-0.045 \pm 0.012 \\
-0.043 \pm 0.012 \\
-0.046 \pm 0.013 \\
-0.066 \pm 0.013\end{array}$ & $\begin{array}{l}-0.044 \pm 0.004 \\
-0.019 \pm 0.005 \\
-0.021 \pm 0.004 \\
-0.006 \pm 0.004 \\
-0.021 \pm 0.004\end{array}$ & $\begin{array}{l}-0.061 \pm 0.003 \\
-0.031 \pm 0.004 \\
-0.034 \pm 0.003 \\
-0.037 \pm 0.003 \\
-0.042 \pm 0.005\end{array}$ \\
\hline $\begin{array}{l}{[\mathrm{La} / \mathrm{Fe}]} \\
{[\mathrm{Ce} / \mathrm{Fe}]} \\
{[\mathrm{Nd} / \mathrm{Fe}]} \\
{[\mathrm{Eu} / \mathrm{Fe}]}\end{array}$ & $\begin{array}{l}0.035 \pm 0.003 \\
0.027 \pm 0.003 \\
0.027 \pm 0.002 \\
0.025 \pm 0.003\end{array}$ & $\begin{array}{l}-0.40 \pm 0.03 \\
-0.31 \pm 0.02 \\
-0.26 \pm 0.02 \\
-0.26 \pm 0.03\end{array}$ & $\begin{array}{l}0.13 \\
0.12 \\
0.10 \\
0.14\end{array}$ & $\begin{array}{l}425 \\
419 \\
427 \\
420\end{array}$ & $\begin{array}{l}0.057 \pm 0.006 \\
0.063 \pm 0.008 \\
0.057 \pm 0.006 \\
0.043 \pm 0.006\end{array}$ & $\begin{array}{r}0.011 \pm 0.011 \\
0.009 \pm 0.012 \\
-0.023 \pm 0.011 \\
-0.007 \pm 0.010\end{array}$ & $\begin{array}{l}0.043 \pm 0.004 \\
0.033 \pm 0.004 \\
0.045 \pm 0.004 \\
0.030 \pm 0.003\end{array}$ & $\begin{array}{l}0.029 \pm 0.003 \\
0.027 \pm 0.002 \\
0.023 \pm 0.002 \\
0.015 \pm 0.004\end{array}$ \\
\hline \multicolumn{9}{|c|}{ as a function of $\log P$} \\
\hline $\begin{array}{l}{[\mathrm{Y} / \mathrm{H}]} \\
{[\mathrm{La} / \mathrm{H}]} \\
{[\mathrm{Ce} / \mathrm{H}]} \\
{[\mathrm{Nd} / \mathrm{H}]} \\
{[\mathrm{Eu} / \mathrm{H}]}\end{array}$ & $\begin{array}{l}0.20 \pm 0.03 \\
0.10 \pm 0.02 \\
0.06 \pm 0.02 \\
0.10 \pm 0.02 \\
0.15 \pm 0.03\end{array}$ & $\begin{array}{l}-0.20 \pm 0.03 \\
-0.13 \pm 0.02 \\
-0.07 \pm 0.02 \\
-0.07 \pm 0.02 \\
-0.11 \pm 0.03\end{array}$ & $\begin{array}{l}0.17 \\
0.14 \\
0.13 \\
0.14 \\
0.17\end{array}$ & $\begin{array}{l}430 \\
424 \\
417 \\
430 \\
418\end{array}$ & $\begin{array}{r}0.28 \pm 0.06 \\
0.06 \pm 0.04 \\
-0.10 \pm 0.07 \\
-0.03 \pm 0.05 \\
0.08 \pm 0.04\end{array}$ & $\begin{array}{l}0.27 \pm 0.07 \\
0.16 \pm 0.07 \\
0.10 \pm 0.07 \\
0.13 \pm 0.07 \\
0.26 \pm 0.08\end{array}$ & $\begin{array}{l}0.12 \pm 0.03 \\
0.04 \pm 0.03 \\
0.00 \pm 0.03 \\
0.04 \pm 0.03 \\
0.09 \pm 0.03\end{array}$ & $\begin{array}{l}0.13 \pm 0.03 \\
0.11 \pm 0.03 \\
0.04 \pm 0.03 \\
0.12 \pm 0.02 \\
0.15 \pm 0.04\end{array}$ \\
\hline$[\mathrm{Ce} / \mathrm{Fe}]$ & $-0.09 \pm 0.02$ & $0.02 \pm 0.02$ & 0.13 & 423 & $-0.55 \pm 0.10$ & $-0.04 \pm 0.07$ & $-0.08 \pm 0.03$ & $-0.09 \pm 0.02$ \\
\hline
\end{tabular}

Notes. ${ }^{(a)}$ In units of dex kpc ${ }^{-1}$ if in function of $R_{\mathrm{G}}$, and dex per logarithmic day if in function of $\log P$. Columns from 2 to 5 shows the results for all the different samples fitted together. We also list the standard deviation $(\sigma)$ of the residuals and the number of data points $(N)$ used in the fit. The slopes using only the stars of our sample (TS: this study) and of previous studies (LEM, LII, and LIII) are shown for comparison.

indicates that young stellar tracers show radial gradients across the Galactic thin disk. A more quantitative discussion concerning the overall behavior will be addressed in a forthcoming paper. Finally, we note that the occurrence of welldefined radial gradients for light $(\mathrm{Y})$ and heavy $s$-process elements ( $\mathrm{La}, \mathrm{Ce}, \mathrm{Nd}$ ) do not support the lack of a radial gradient for $\mathrm{Ba}$ as recently suggested by Andrievsky et al. (2014) and by Martin et al. (2015). The quoted authors used high-resolution spectra for a sizable sample of inner and outer disk classical Cepheids and take NLTE effects into account. However, Ba abundances in classical Cepheids are affected by severe limits. In particular, Luck (2014) noted that strong BaII lines are affected by line-formation effects, while Andrievsky et al. (2013) discussed physical and atomic (isotopic shifts) effects in detail. The reason for the lack of a Ba gradient remains unclear.

ii) - Slopes - The slopes are quite similar and are on average $-0.025 \pm 0.004 \mathrm{dex} \mathrm{kpc}^{-1}$ for $\mathrm{La}, \mathrm{Ce}, \mathrm{Nd}$, and $\mathrm{Eu}$. The only exception is $\mathrm{Y}$, for which the slope is more than a factor of two steeper $\left(-0.053 \pm 0.003 \mathrm{dex} \mathrm{kpc}^{-1}\right)$. The current slopes agree quite well, within the errors, with similar estimates available in the literature. We found that the slopes range from $-0.053 \pm 0.003 \mathrm{dex} \mathrm{kpc}^{-1}$ for $[\mathrm{Y} / \mathrm{H}]$ to $-0.020 \pm 0.003$ for $[\mathrm{La} / \mathrm{H}]$. The slopes estimated by LII+LIII for the same elements range from $-0.061 \pm 0.003$ to $-0.019 \pm 0.005 \mathrm{dex} \mathrm{kpc}^{-1}$, while those estimated by LEM range from $-0.062 \pm 0.012$ to $-0.045 \pm 0.012 \mathrm{dex} \mathrm{kpc}^{-1}$. This last estimation is slightly steeper and the difference might be due to the limited range in Galactocentric distances covered by their Cepheid sample. The main difference in the comparison with similar estimates available in literature is for Nd. Indeed, LII found a flat distribution across the thin disk. We performed several tests using different cuts in Galactocentric distance and in sample size and we found that the slope is solid within the current uncertainties (see labeled error bars). Moreover, the standard deviation of the
Nd gradient is the smallest among those investigated (for details on the slopes of the available data sets, see Cols. 6-9 in Table 6).

iii) - Spread - The spread of the individual abundances attains similar values across the thin disk. The outermost disk regions are an exception since the spread increases for $R_{\mathrm{G}}$ larger than $13 \mathrm{kpc}$. The neutron capture elements display the same trend of iron and $\alpha$-element abundances. Among the investigated elements, $\mathrm{Y}$ once again seems to be an exception, since the spread is homogeneous over the range of Galactocentric distances covered by the current sample.

iv) - Comparison with theory - Our results for $\mathrm{La} \quad\left(-0.020 \pm 0.003 \mathrm{dex} \mathrm{kpc}^{-1}\right)$ and for $\mathrm{Eu}$ $\left(-0.030 \pm 0.004 \mathrm{dex} \mathrm{kpc}^{-1}\right)$ agree quite well with theoretical predictions by Cescutti et al. (2007) for Galactocentric distances covering the entire thin disk $\left(4 \leq R_{\mathrm{G}} \leq 22 \mathrm{kpc}\right)$. They found a slope of $-0.021 \mathrm{dex} \mathrm{kpc}^{-1}$ for $\mathrm{La}$ and of $-0.030 \mathrm{dex} \mathrm{kpc}^{-1}$ for Eu. The predicted slopes become steeper for Galactocentric distances shorter than $14 \mathrm{kpc}$ and shallower for distances larger than $16 \mathrm{kpc}$ (see their Table 5). Predictions for the other $s$-process elements are not available. We note that the observed slope for $\mathrm{Y}$ $\left(-0.053 \pm 0.003 \mathrm{dex} \mathrm{kpc}^{-1}\right)$ is similar to the predicted slopes for iron and iron-group elements in the Galactocentric range between 4 and $14 \mathrm{kpc}$.

\subsection{Comparisons with independent radial gradients}

To further constrain the plausibility of the above radial gradients, Fig. 3 shows the comparison between Cepheid gradients and radial gradients of neutron-capture elements of Galactic field stars. The abundances of Y, Ce, Nd, and Eu for $181 \mathrm{~F}$ - and G-type dwarf stars provided by Reddy et al. (2003, hereafter R03) are plotted in this figure. The $\mathrm{La}$ and $\mathrm{Eu}$ abundances for 159 dwarf and giant stars were provided by Simmerer et al. (2004, hereafter S04). Their abundances were rescaled to the abundances of the 
R. da Silva et al.: Neutron-capture elements across the Galactic thin disk using Cepheids

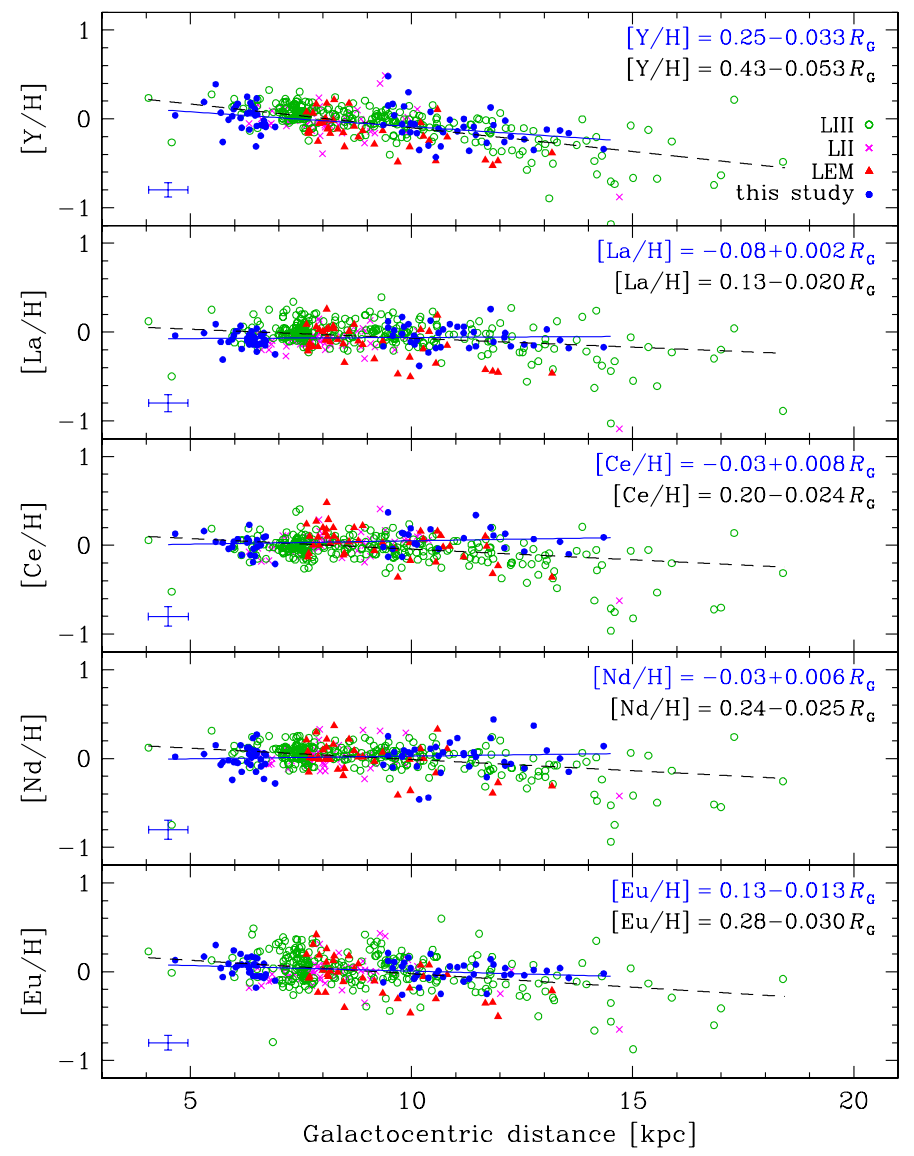

Fig. 2. Abundances of neutron-capture elements as a function of $R_{\mathrm{G}}$. Our results (filled blue circles) are compared with those of Luck et al. (2011, LII, magenta crosses), Luck \& Lambert (2011, LIII open green circles), and Lemasle et al. (2013, LEM, red triangles). The blue solid line shows the linear regression of our Cepheid sample, while the black dashed line the linear regression of the entire Cepheid sample. The blue error bars give the mean spectroscopic error of the current sample. The abundances available in the literature have similar errors.

solar mixture adopted in the current investigation (Grevesse et al. 1996). Moreover, to overcome possible differences between the Cepheids and field stars concerning either the different diagnostics adopted to determine distances or the use of different spectral lines when plotting these data, we adopted the zero-points of our gradients at the solar Galactocentric distance. We note that when dealing with the S04 data, we only selected the more metal-rich stars $([\mathrm{Fe} / \mathrm{H}]>-1.0)$ to be more consistent with the metallicity range of the current Cepheids. The figure shows that the radial gradients of the five neutron-capture elements based on the Cepheids agree quite well with the abundances for field dwarf stars in the Galactic thin disk. The fact that the giants in the S04 sample covers only a limited range of Galactocentric distances across the solar circle does not allow us to constrain the radial gradient.

In Fig. 3 we also plot the $\mathrm{Y}$ abundances recently provided by Origlia et al. (2013, hereafter O13) for three red supergiant (RSG) stars in the Scutum cluster. They used high-resolution $(R \sim 50000)$ NIR $(Y, J, H, K)$ spectra collected with GIANO at the Telescopio Nazionale Galileo (TNG). The comparison further supports previous results by Bono et al. (2015) and G15 concerning the underabundance of iron and $\alpha$-elements in blue and red supergiants located either in the near end of the Galactic bar or in the Galactic center. The Y abundances display the

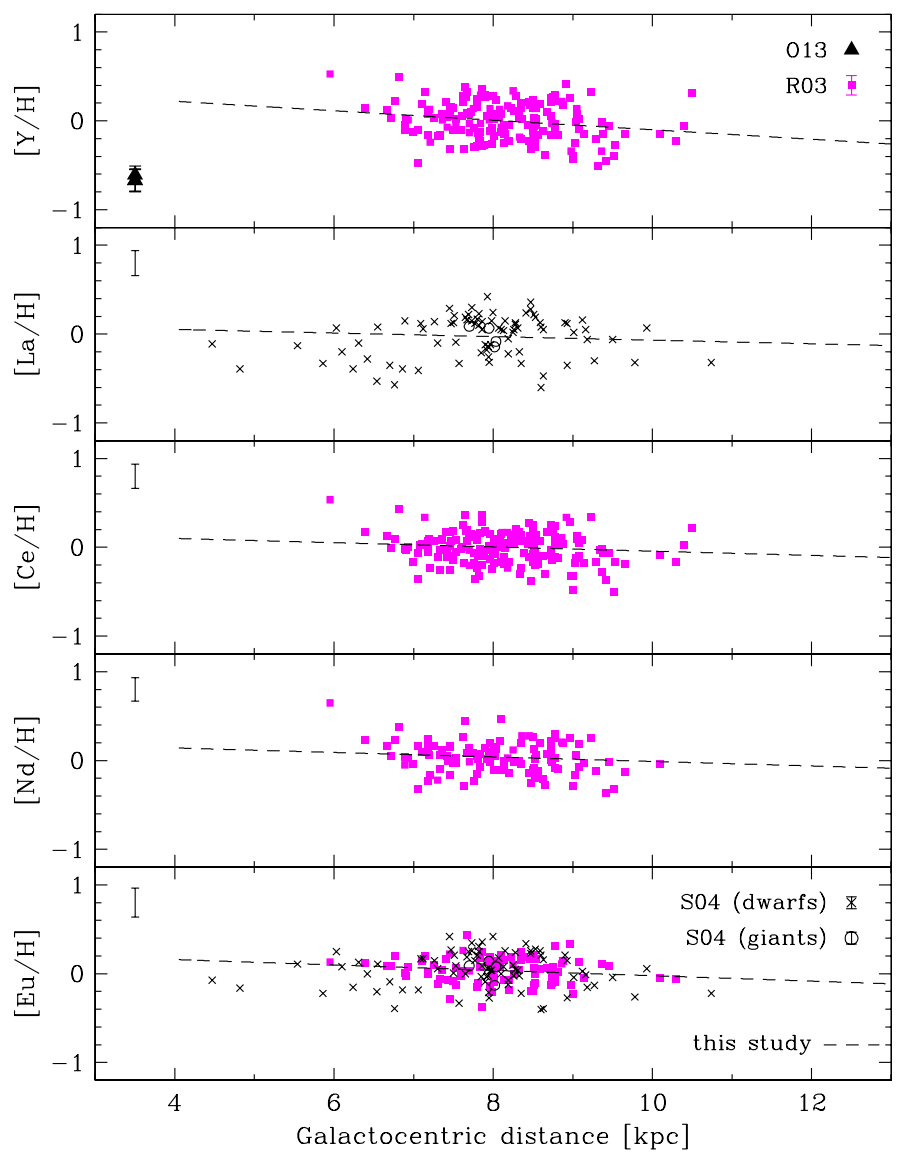

Fig. 3. Abundances of neutron-capture elements as a function of $R_{\mathrm{G}}$. The radial gradients we derived for Cepheid stars (dashed line) are compared with field dwarfs analyzed by Reddy et al. (2003, R03, magenta squares) and with field dwarfs (crosses) and giants (open circles) analyzed by Simmerer et al. (2004, S04). From the latter only stars with $[\mathrm{Fe} / \mathrm{H}]>-1.0$ are plotted. RSGs in the Scutum cluster analyzed by Origlia et al. (2013, O13, triangles) are also shown.

same underabundance when compared with similar abundances of classical Cepheids located in the inner edge of the Galactic thin disk.

\subsection{Age dependence of the [neutron-capture/H] ratios}

The results concerning the abundance gradients discussed in the above sections use the Galactocentric distance as independent variable. However, classical Cepheids when compared with other stellar tracers have the particular advantage that their pulsation period is highly anticorrelated with their individual ages (Bono et al. 2005, G15). The typical pulsation age of shortperiod ( $P \sim 1.0-1.5$ days) Cepheids is indeed on the order of $200 \mathrm{Myr}$, while for long-period ( $P \sim 100$ days) Cepheids it is on the order of $10 \mathrm{Myr}$. The exact range in age depends on the chemical composition and on the adopted evolutionary framework (see Anderson et al. 2015 and Tables 4 and 5 in Bono et al. 2005). This provides the unique opportunity of constraining the chemical enrichment history of the thin disk during the last 300 Myr (G14, G15).

To constrain the age dependence of the metallicity gradients, Fig. 4 shows the same elemental abundances plotted in Fig. 2, but as a function of the logarithmic period. Data plotted in this figure show that the investigated neutron-capture elements display well-defined positive gradients as a function of the pulsation 


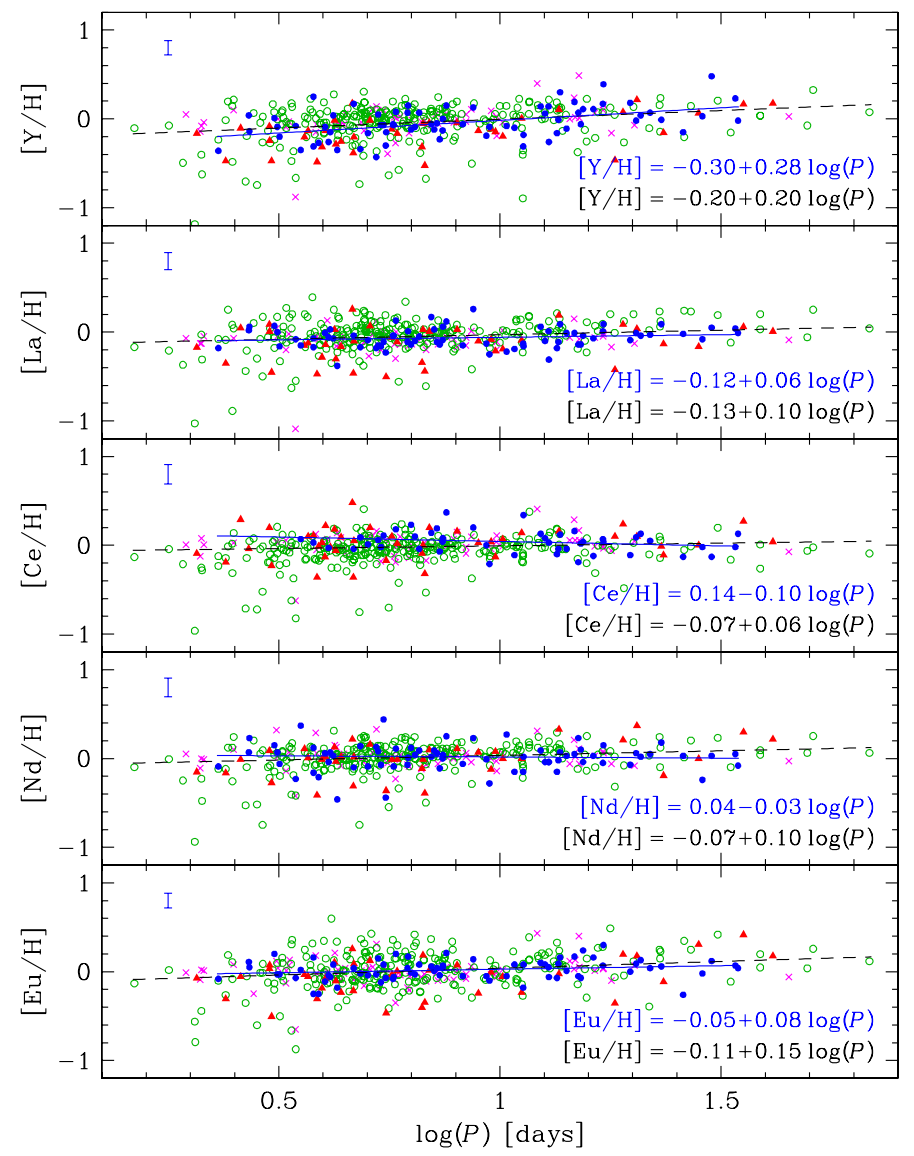

Fig. 4. Abundances of neutron-capture elements as a function of the logarithmic pulsation period. Symbols and colors are the same as in Fig. 2.

period. The $\alpha$-elements $(\mathrm{Mg}, \mathrm{Si}, \mathrm{Ca})$ and the light elements $(\mathrm{Na}$, Al) investigated by G15 show similar trends, but the current slopes are on average shallower. The slopes of three out of the four $s$-process elements ( $\mathrm{La}, \mathrm{Ce}, \mathrm{Nd}$ ) are equal or smaller than 0.10 dex per logarithmic day; however, Y (s element) and Eu ( $r$ element) display steeper slopes $(0.20$ and 0.15 dex per logarithmic day, respectively).

The above empirical evidence indicates that the elements that are more typically associated with explosive nucleosynthesis $(\mathrm{Si}$, $\mathrm{Ca}, \mathrm{Eu})$ display age gradients ranging from $0.09(\mathrm{Ca})$ to 0.15 ( $\mathrm{Si}, \mathrm{Eu})$ dex per logarithmic day. On the other hand, Y shows a slope $(0.20 \pm 0.03$ dex per logarithmic day $)$ that is at least a factor of two larger than the other $s$-process elements with similar s-fraction abundances ( $\mathrm{La}, \mathrm{Ce}$ ). In this context it is worth mentioning that the $60-70 \%$ of $\mathrm{Y}$ is produced in the main $s$-process, while $5-10 \%$ comes from $r$-process and the remaining from the weak component. However, the significant difference in the Y slope when compared with the other $s$-process elements could suggest a larger contribution either from the $r$ - and/or from the s-weak component.

To take the above empirical evidence into account we could also use plain stellar evolutionary arguments. We start from the evidence that Cepheid stellar masses range, according to chemical composition, from 3.0-3.5 $M_{\odot}$ to $10-12 M_{\odot}$ (Bono et al. 2010). This means that a significant fraction of Cepheids evolve into the AGB phase. The difference in evolutionary time between the end of the so-called blue loop and the beginning of the AGB phase is negligible when compared with $\mathrm{H}$ and He burning phases. This means that Cepheids and AGB stars with stellar

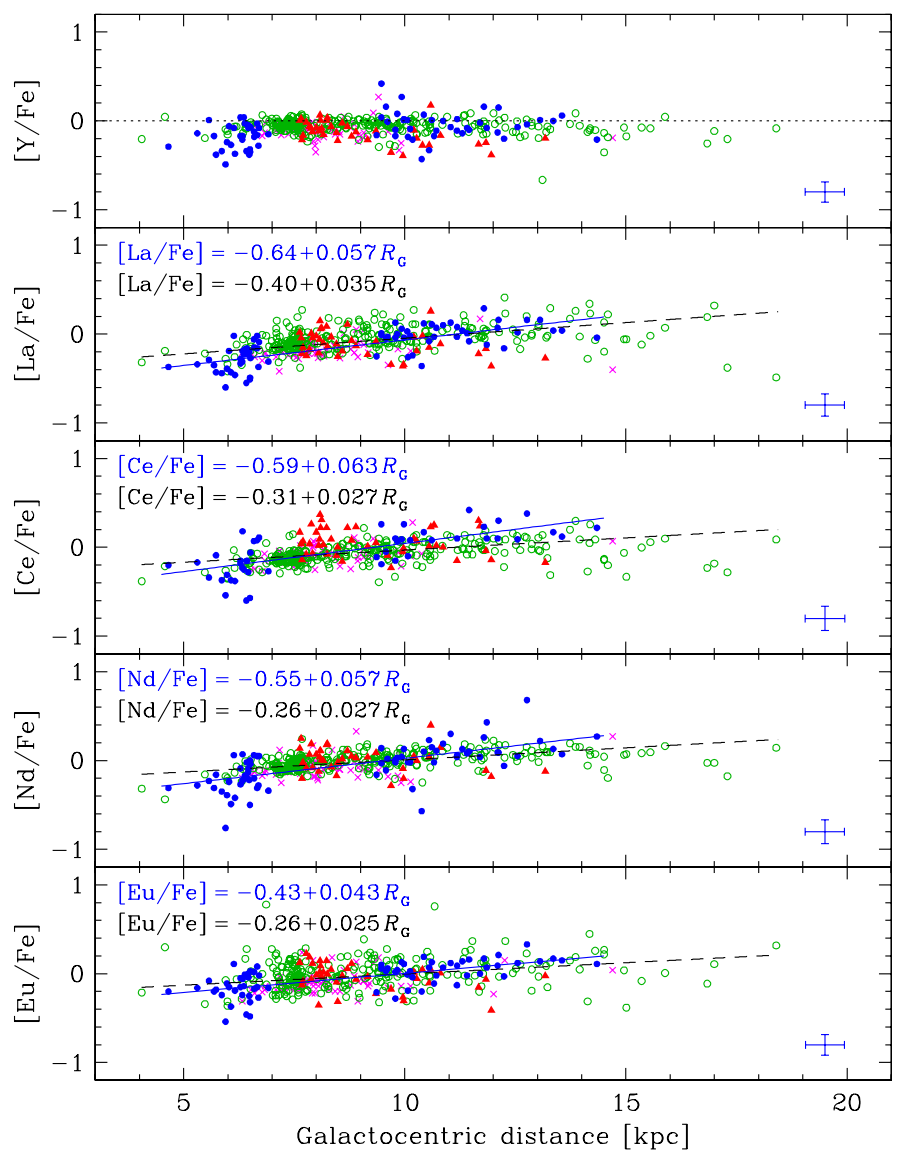

Fig. 5. Same as Fig. 2, but the abundances are scaled to iron. The dotted line indicates the positions of solar abundance ratios.

masses ranging from $\sim 3$ to $\sim 6 M_{\odot}$ evolve with similar evolutionary lifetimes. The current theoretical predictions indicate that intermediate-mass AGB stars in the quoted mass regime mainly produce light $s$-process (ls) elements (such as Y), while most of the heavy $s$-process (hs) elements (such as La) are mainly produced in low-mass $\left(M<3 M_{\odot}\right)$ AGB stars. To further constrain this effect, we mention that an AGB star of $6 M_{\odot}$ produces roughly $1 / 3$ of the $\mathrm{Y}$, but only $1 / 7$ of the La produced by a $3 M_{\odot}$ (Cristallo et al. 2015a). This would imply that for younger Cepheids $\mathrm{Y}$ is a good tracer of the recent chemical enrichment of intermediate-mass AGB stars. The same outcome applies for the slope of Eu since this element is mainly produced in stellar structures that are either coeval or even younger than Cepheids. It goes without saying that the quoted scenario is qualitative and more detailed calculations based on chemo-dynamical models are required to constrain the anticorrelation between s- and $r$-process elements with age.

\subsection{Radial gradient of [neutron-capture/Fe]}

Figure 5 shows the radial gradients of the abundance ratios scaled to iron. Similar radial gradients for the $\alpha$-elements were recently investigated by $\mathrm{G} 15$. The test was motivated by the similarity in the slope of $[\mathrm{Fe} / \mathrm{H}]$ and $[\alpha / \mathrm{H}]$ ratios. They found that the slopes of $[\alpha / \mathrm{Fe}]$ ratios as a function of the Galactocentric distance are typically smaller than $0.018 \pm 0.002 \mathrm{dex} \mathrm{kpc}^{-1}$. The conclusion for the quoted elements was that they show, on average, quite flat distributions across the entire thin disk. 
Data plotted in Fig. 5 display a different empirical scenario for neutron-capture elements. The $s$ - (La, Ce, Nd) and $r$ - (Eu) process elements display slopes that are, on average, a factor of two larger than $[\alpha / \mathrm{Fe}]$ ratios. The only element to show a flat distribution over the entire disk is Y. The above evidence suggests that the steady enhancement in four out of the five neutroncapture elements investigated is mainly caused by the slopes of $\mathrm{La}, \mathrm{Ce}, \mathrm{Nd}$, and Eu radial gradients: they are at least a factor of two smaller than the iron slope $(-0.060 \pm 0.002 \mathrm{dex} \mathrm{kpc}-1)$. The $[\mathrm{Y} / \mathrm{Fe}]$ ratio is flat because the slope of the $\mathrm{Y}$ gradient $\left(-0.053 \pm 0.003 \mathrm{dex} \mathrm{kpc}^{-1}\right)$ is quite similar to the value for iron.

The above findings indicate that the chemical enrichment history of $\mathrm{La}, \mathrm{Ce}, \mathrm{Nd}$, and $\mathrm{Eu}$ across the Galactic thin disk is quite different when compared with $\alpha$-elements and iron. Although Y is considered mainly a s-process element, its abundance ratios appear to be more similar to iron and to $\alpha$-elements than to the other neutron-capture elements. It is worth mentioning that the spread in [element/Fe] of the five investigated elements appears to be quite constant when moving from the inner to the outer disk (see Fig. 5). There is also a mild evidence of a flattening in the above ratios towards the outer disk. The radial gradients based on the current sample are steeper than the slopes based on the entire sample. The difference is mainly due to the limited Galactocentric distance covered by our sample. However, the number of Cepheids with Galactocentric distance larger than $13 \mathrm{kpc}$ is limited, and new identifications of classical Cepheids in the outer disk are required to further constrain the quoted trends (see also G15). In this context, it is worth mentioning that Gaia will play a crucial role, since detailed calculations indicate that the number of Galactic Cepheids will increase by at least one order of magnitude (Bono 2003; Windmark et al. 2011).

\subsection{Comparisons with independent radial gradients}

To validate the new slopes of the [neutron-capture/Fe] radial gradients, Fig. 6 shows a comparison with similar data available in the literature. The colored symbols denote the same field dwarf and giant stars plotted in Fig. 3. We note that the abundance ratios plotted in this figure were scaled both in iron and in neutron-capture element abundances. The flatness of the [neutron-capture/Fe] ratios for $\mathrm{Y}$ and the increasing trends for $\mathrm{La}, \mathrm{Ce}, \mathrm{Nd}$, and $\mathrm{Eu}$ are quite similar to the results based on the entire Cepheid sample.

This evidence further supports our working hypothesis that during the last 300 Myr neutron-capture elements - with the exception of $\mathrm{Y}$ - experienced a different chemical enrichment history from iron and $\alpha$-elements. The current predictions concerning the chemical enrichment of AGB stars indicate that 1s elements (such as Y) are mainly synthesized in the more metalrich $([\mathrm{Fe} / \mathrm{H}]>-0.6)$ regime, while the hs elements (such as La) are more favored in the metal-intermediate regime (see also Sect. 4). The quoted theoretical framework supports the mild enhancement in hs elements when moving from the inner (more metal-rich) to the outer (more metal-poor) Galactic thin disk. On the other hand, the lack of a clear trend in the [Y/ Fe] abundance ratio indicates a substantial balance across the entire disk. However, the most metal-rich $([\mathrm{Fe} / \mathrm{H}] \sim 0.4-0.5)$ Cepheids in our sample that are located in the inner disk $\left(5 \leq R_{\mathrm{G}} \leq 7 \mathrm{kpc}\right)$ show a downturn in $[\mathrm{Y} / \mathrm{Fe}]$, suggesting an underabundance of $\mathrm{Y}$ at super-solar iron abundance. This finding further supports a similar trend in $[\mathrm{Y} / \mathrm{Fe}]$ abundances provided by Feltzing \& Gustafsson (1998, hereafter FG98) using high-resolution spectra for 47 dwarf stars with super-solar iron abundance (see their

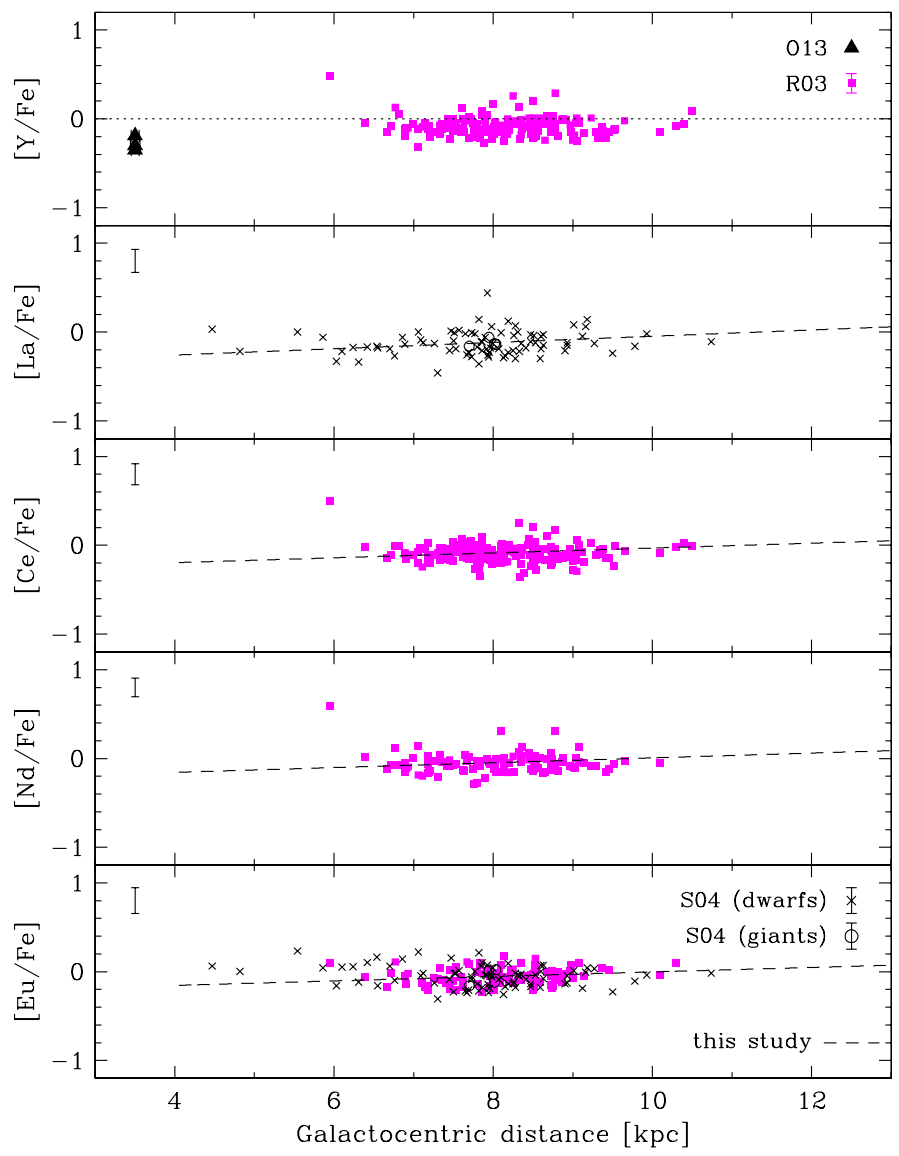

Fig. 6. Same as Fig. 3, but the abundances are scaled to iron. The dotted line indicates the positions of solar abundance ratios.

Fig. 22 and Sect. 6.13). However, the presence of a mild enhancement of Eu in the outer disk is even more compelling, since this is considered a solid $r$-process element mainly produced by the same stellar masses producing $\alpha$-elements.

We also note that the reduced spread of the above elements at fixed $R_{\mathrm{G}}$ distance also suggests an almost homogeneous spatial enrichment across the four Galactic quadrants. This is also interesting evidence worth investigating in more detail since AGB stars can have both intermediate-age (1-9 Gyr) and old ( 10 Gyr) progenitors.

\subsection{Age dependence of the [neutron-capture/Fe] ratios}

To constrain the age dependence of the [neutron-capture/Fe] abundance ratios, Fig. 7 shows the same elemental abundances plotted in Fig. 5, but as a function of the logarithmic period. A glance at the data plotted in this figure shows that the ratios are approximately constant over the entire period range. The only exception is $\mathrm{Ce}$, which shows a mild negative gradient $(-0.09 \pm 0.02$ dex per logarithmic day). Similar trends are also shown by light and $\alpha$-elements. Indeed, Ca showed (see Fig. 5 in G15) a negative gradient $(-0.11 \pm 0.02$ dex per logarithmic day), while the others either a mild gradient $(\mathrm{Al}, \mathrm{Si})$ or a flat distribution $(\mathrm{Na}, \mathrm{Mg})$. The flattening of the $s$-process elements is once again an interesting finding, since it suggests that s elements and iron enrichment across the Galactic thin disk have been homogenous over a broad range of ages. The zero-point, the slope, their uncertainties, and the standard deviation of the Ce gradient are listed in the bottom line of Table 6 . 


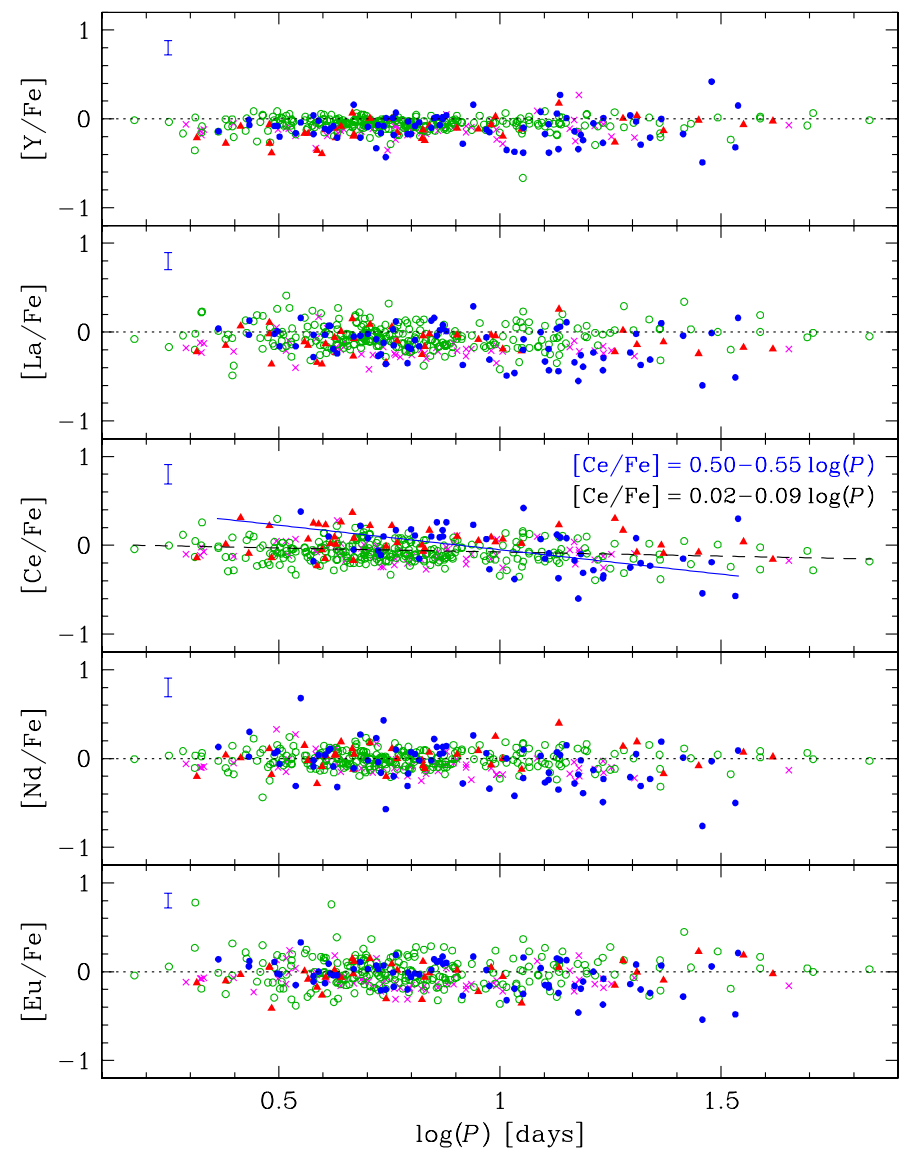

Fig. 7. Same as Fig. 4, but the abundances are scaled to iron. The dotted lines indicate the positions of solar abundance ratios. Symbols and colors are the same as in Fig. 2.

\section{Neutron-capture element relative abundances}

\subsection{Metallicity dependence of the [neutron-capture/Fe] ratios}

The comparison with abundances of neutron-capture elements available in the literature discussed in the above sections were limited to the data sets for which individual Galactocentric distances were also available. In this section we perform the comparison only using elemental abundances. In particular, we selected i) Y, Ce, Nd, and Eu abundances of F- and G-type field dwarf stars provided by R03 (181 objects); ii) Y and Eu abundances of F- and G-type field dwarf stars estimated by Bensby et al. (2005, hereafter B05, 102 objects), including both thin and thick disk stars; iii) $\mathrm{La}$ and Eu abundances of field dwarf and giant stars provided by S04 (159 objects); iv) Y abundances of field dwarfs analyzed by Edvardsson et al. (1993, hereafter E93, 157 objects); v) Y, La, Nd, and Eu abundances of 47 supermetal-rich field dwarfs by FG98; and vi) Y abundances of three RSG stars in the Scutum cluster measured by O13.

Figure 8 shows the comparison between Cepheid [neutroncapture/Fe] abundance ratios with the quoted data sets. We applied a shift in the abundances by FG98, R03, and B05 in order to put them on the same scale as our data at solar metallicity. Data plotted in this figure show that the agreement between Cepheids and both field dwarfs and field giants in the Galactic disk is quite good over the entire metallicity range covered by the above samples. The trends are flat across solar iron abundances and display a modest abundance dispersion. Moreover, there is a clear decrease in the [element/Fe] ratios in the super

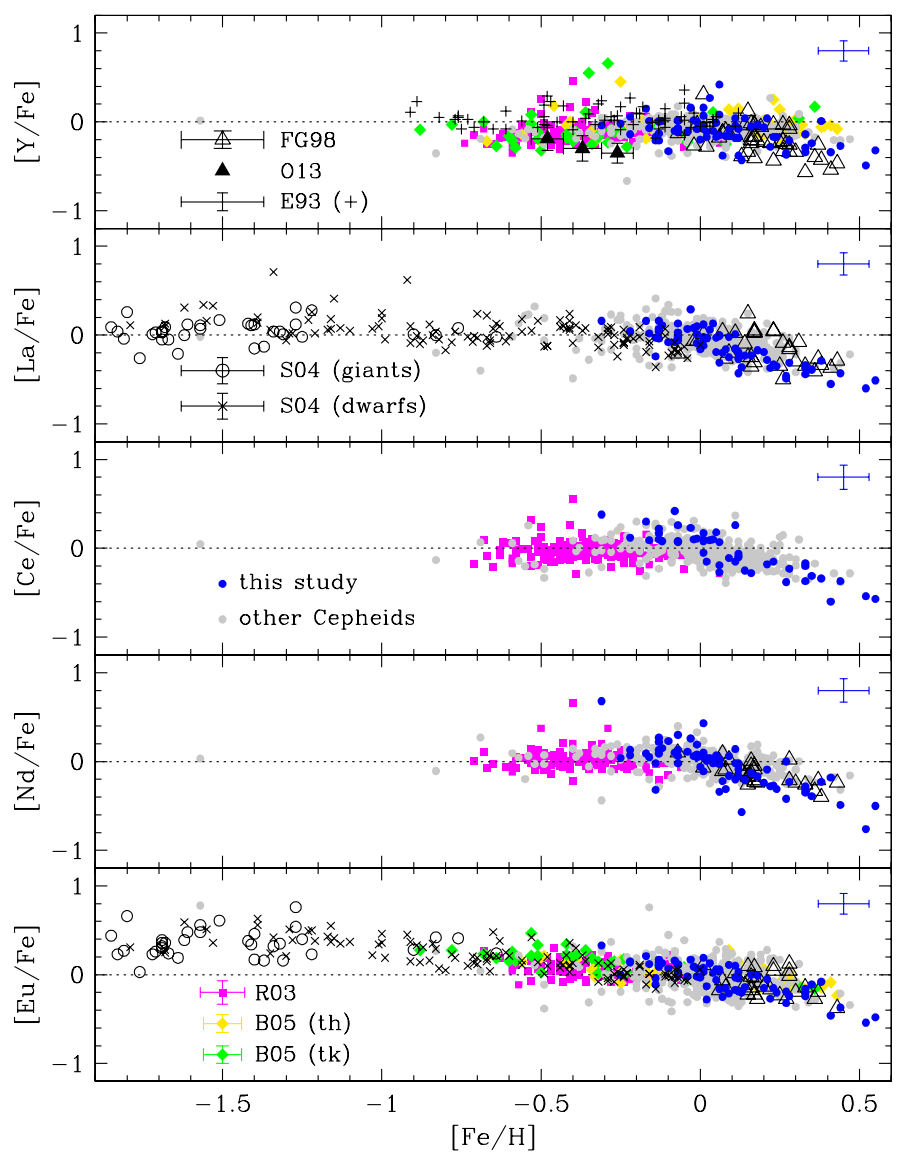

Fig. 8. Abundances of neutron-capture elements as a function of the metallicity. Cepheid stars (filled circles) are compared with field dwarfs from the thin disk analyzed by R03 (magenta squares), from the thin disk (yellow diamonds) and thick disk (green diamonds) in Bensby et al. (2005, B05); compared with field dwarfs (crosses) and giants (open circles) in S04; and compared with field dwarfs in Edvardsson et al. (1993, E93, pluses) and in Feltzing \& Gustafsson (1998, FG98, open triangles). RSG in the Scutum cluster analyzed by $\mathrm{O} 13$ (filled triangles) are also shown. The dotted lines indicate the positions of solar abundance ratios.

metal-rich regime $([\mathrm{Fe} / \mathrm{H}]>0.2)$, thus suggesting a significant contribution in this iron regime from $\mathrm{SNe}$ type Ia ejecta.

The $[\mathrm{Y} / \mathrm{Fe}]$ ratios have, once again, a different trend: it is underabundant and almost constant over the entire metallicity range (see the top panel of Fig. 8). This trend is supported by field dwarfs available in the literature, though for the supermetal-rich stars provided by FG98 the trend seems to be slightly steeper. The three RSGs observed by $\mathrm{O} 13$ also appear, within the errors, similar to the other field disk stars.

The $[\mathrm{La} / \mathrm{Fe}]$ abundance ratio shows a steady enhancement when moving from the metal-rich into the metal-poor regime. In the paper by M13 the authors suggested that this trend is mainly caused by the metallicity dependence in the production of the neutron-capture $s$-process elements (see also Gallino et al. 1998; Busso et al. 1999). The above ratio approaches solar values for $[\mathrm{Fe} / \mathrm{H}] \sim-0.2$ and attains a constant value in the more metal-poor regime, thus suggesting no dependence on iron in this metallicity range. The trend in the metal-rich regime $([\mathrm{Fe} / \mathrm{H}] \geq 0)$ is similar to those for $\mathrm{Ce}$ and $\mathrm{Nd}$, i.e., it is underabundant by roughly 0.5 dex for $[\mathrm{Fe} / \mathrm{H}] \sim 0.5$. The quoted trend is quite evident for $\mathrm{La}, \mathrm{Ce}$, and $\mathrm{Nd}$, as well as for Eu. The Y in the metal-rich regime shows a similar trend, but the underabundance is lower than the above-mentioned elements. 

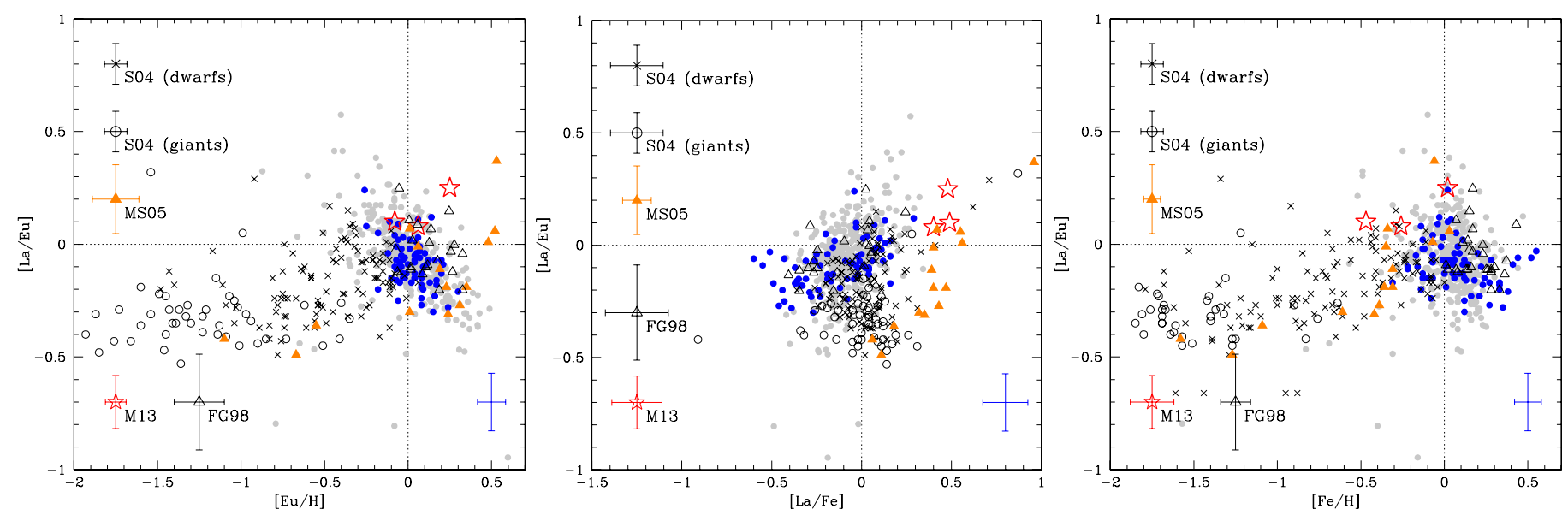

Fig. 9. Abundance ratios between $\mathrm{La}$ and $\mathrm{Eu}$ as a function of $[\mathrm{Eu} / \mathrm{H}]($ left panel), $[\mathrm{La} / \mathrm{Fe}]$ (middle panel), and $[\mathrm{Fe} / \mathrm{H}]$ (right panel). Cepheid stars (filled circles) are compared with Galactic field dwarfs (crosses) and giants (open circles) by S04, with field dwarfs by FG98 (open triangles), and with Sgr field stars studied by McWilliam \& Smecker-Hane (2005, MS05, filled triangles) and by McWilliam et al. (2013, M13, red stars).

The $[\mathrm{Eu} / \mathrm{Fe}]$ abundance ratio shows a different trend. The enhancement is steady over the entire metallicity range covered by Cepheids and by S04 sample. This evidence suggests a strong anticorrelation with iron concerning the Eu production. It is worth mentioning that data plotted in the bottom panel of this figure further support the contribution of SNe type Ia to the iron abundance. The steady decrease in $[\mathrm{Eu} / \mathrm{Fe}]$ abundance ratio can be explained as a steady increase in iron abundance and a marginal, if any, production of Eu. This trend fully supports early results from FG98 for field super-metal-rich dwarfs.

Finally, we also note that the spread in s elements and in Eu is constant over the metallicity range covered by the current samples. There is solid empirical evidence that the spread in Eu increases in the most metal-poor regime, for $[\mathrm{Fe} / \mathrm{H}] \lesssim-2.0$ (Cescutti et al. 2006), but we still lack a detailed quantitative explanation of the observed trend.

\subsection{The $[\mathrm{La} / \mathrm{Eu}]$ and $[\mathrm{Y} / \mathrm{Eu}]$ abundance ratios}

The left panel of Fig. 9 shows the s-to-r abundance ratio $[\mathrm{La} / \mathrm{Eu}]$ vs. the $[\mathrm{Eu} / \mathrm{H}]$ abundance. We note that the abundances derived by S04, McWilliam \& Smecker-Hane (2005, hereafter MS05), and M13 were only rescaled to take into account the solar mixture adopted in the current investigation (Grevesse et al. 1996). We also note that we plotted the [La/Eu] ratio vs. the $[\mathrm{Eu} / \mathrm{H}]$ abundance to separate the role played by pure explosive nucleosynthesis of iron in SNe type Ia and type II from the neutron capture enrichments. The Cepheids in this plane show a well-defined anticorrelation. The $[\mathrm{La} / \mathrm{Eu}]$ ratio, when moving from the most Eu-rich to the most Eu-poor stars, increases by almost one dex. The Cepheid abundance ratio becomes even more compelling in comparison with the field giant and dwarf star values provided by S04. This sample shows an almost constant ratio over a broad range of Eu abundances and a mild increase in the approach to solar Eu abundances. The $[\mathrm{La} / \mathrm{Eu}]$ abundance ratios provided by M13 and by MS05 for Sagittarius RGs show a similar distribution, but three stars display large Eu abundances. Two of the three display a solar ratio, while the third is 0.4 dex enhanced in La.

Data plotted in the middle panel of Fig. 9 shows the same abundance ratios, but versus the $[\mathrm{La} / \mathrm{Fe}]$ ratio. The empirical scenario becomes clearer, and indeed we found that RGs in Sagittarius are systematically more enhanced in La when compared with Galactic thin disk stellar population. Indeed, only a few Sagittarius stars are located in the same region covered by thin disk stars. We note that a similar enhancement in La has also been found in several dwarf spheroidal galaxies (Shetrone et al. 2003; Geisler et al. 2005; Pompeia et al. 2008; Letarte et al. 2010; Lemasle et al. 2014), thus suggesting that the above plane is a good diagnostic to identify relic stars of dwarf galaxies that have been accreted by the Milky Way.

The right panel of Fig. 9 shows the same abundance ratio, but versus the iron abundance. The trend is quite similar to the left panel of the same figure. However, Cepheids and field stars display a larger spread in iron at fixed [La/Eu]. The RGs in Sagittarius (MS05, M13) display a trend similar to the Galactic stars, and for $[\mathrm{Fe} / \mathrm{H}]>-0.3$ it is also similar to Galactic Cepheids. The above evidence indicates that s- and $r$-process elements in the Galactic thin disk have similar enrichment histories in the metal-poor regime $([\mathrm{Fe} / \mathrm{H}] \leq-0.3)$. In the more metalrich regime, the same ratio shows a well-defined anticorrelation with iron and with Eu abundances.

To further constrain the ratio between $s$ - and $r$-process elements we also investigate the abundance ratio between a light s-element (Y) and Eu. The left panel of Fig. 10 shows the $[\mathrm{Y} / \mathrm{Eu}]$ vs. $[\mathrm{Eu} / \mathrm{H}]$ plane. The distribution of Cepheids in this plane is quite different from the $[\mathrm{La} / \mathrm{Eu}]$ vs. $[\mathrm{Eu} / \mathrm{H}]$ plane. Cepheids show a larger dispersion over the entire metallicity range they cover, and there is no clear evidence of an anticorrelation with the europium content. On the other hand, field stars show a mild evidence of a correlation with Eu abundance when moving from the Eu-intermediate into the more Eu-rich regime.

Data plotted in the middle panel of Fig. 10 shows a welldefined correlation between $[\mathrm{Y} / \mathrm{Eu}]$ and $[\mathrm{Y} / \mathrm{Fe}]$. This finding together with the constant value of the $[\mathrm{Y} / \mathrm{Fe}]$ as a function of both Cepheid ages and iron abundance suggests a different enrichment history between $\mathrm{Y}$ and $\mathrm{Eu}$, but also a difference between light (Y) and heavy (La) $s$-process elements. We note that the three Sagittarius RGs attain in this plane the lowest values, thus suggesting that they are quite $\mathrm{Y}$ poor when compared with field Galactic stars.

The right panel of Fig. 10 shows the same data, but in the [Y/Eu] vs. iron abundance. The bulk of the data seems to suggest a correlation between the s-to-r abundance ratio and iron content. In this context it is worth mentioning that for iron abundances more metal-poor than the Sun there is slight evidence of a 

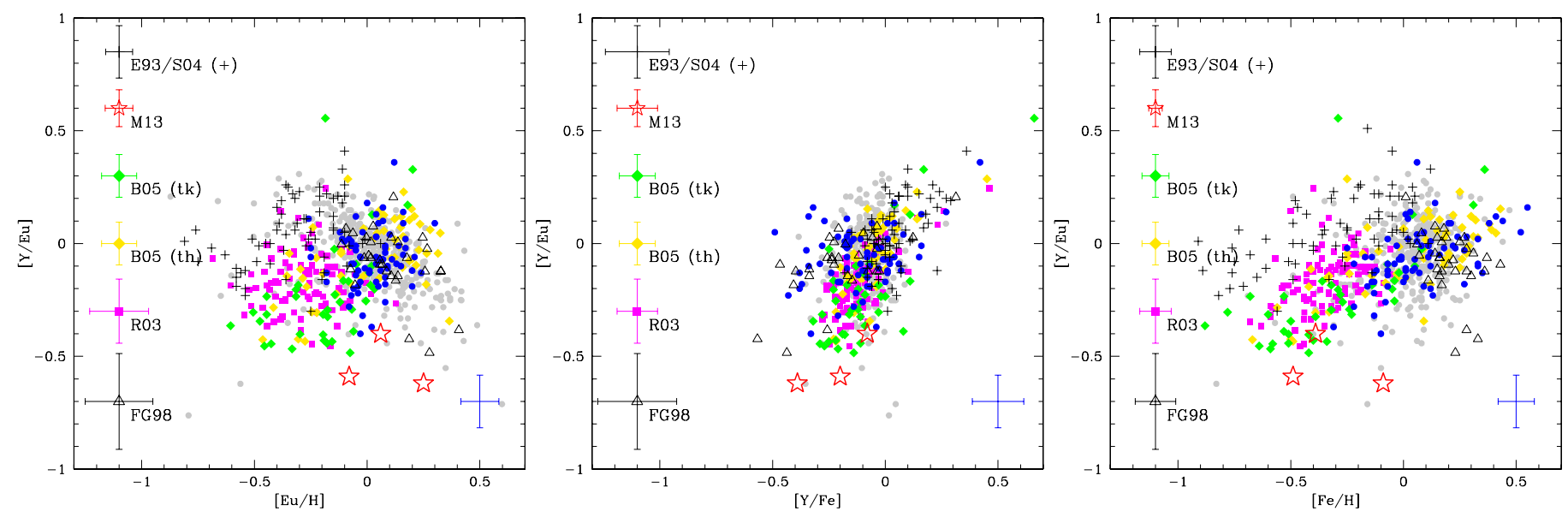

Fig. 10. Same as in Fig. 9, but for Y and Eu ratios. Galactic field dwarfs by E93 and S04 (pluses), field dwarfs from the thin disk by R03 (magenta squares), and field dwarfs from the thin (yellow diamonds) and thick (green diamonds) disks by B05 are also shown.

possible dichotomous distribution. In particular, field dwarf stars associated with the Galactic thick disk provided by B05 display, at fixed iron content, lower $[\mathrm{Y} / \mathrm{Eu}]$ abundance ratios. The difference with similar abundances provided by E93 plus S04 and by R03 is slightly larger than one sigma and needs to be further investigated with a larger homogeneous sample.

\subsection{The $[\mathrm{La} / \mathrm{Y}]$ abundance ratio}

Figure 11 shows the ratio between a heavy (La) and a light (Y) neutron-capture element. Such a ratio is a good diagnostic for the $s$-process index $[\mathrm{hs} / \mathrm{ls}]$, i.e., the ratio between the heavy $s$-process elements and the light ones. The quoted ratio and its dependence on the metallicity are solid tracers of the role played by AGB stars in the chemical enrichment (Gallino et al. 1998; Busso et al. 1999, 2001; Cristallo et al. 2009). The production of hs elements (such as La) is favored in the metalintermediate regime $([\mathrm{Fe} / \mathrm{H}] \sim-0.6)$, while in the more metalrich regime ls elements (such as Y) are mostly synthesized. Therefore, the ratio [hs/ls] is expected to be underabundant in the metal-rich regime and enhanced in the more metal-poor regime. Our Cepheid data in the left panel of this figure display, in agreement with theoretical predictions (Cristallo et al. 2009, 2011, 2015 b), a well-defined anticorrelation between the $[\mathrm{La} / \mathrm{Y}]$ ratio and the Y abundance. The Galactic field stars measured by E93 and S04 display a wide spread, at fixed Y abundance, but the trend is similar. Interestingly enough, we found that Sagittarius RGs - provided by MS05, M13, and Sbordone et al. (2007, hereafter S07) - display two distinctive features: i) a strong enhancement in La with a marginal overlap with Galactic stars and ii) a spread in $[\mathrm{La} / \mathrm{Y}]$ abundance ratio that is significantly larger than Galactic stars. Thus suggesting that the enrichment of neutroncapture elements in Sagittarius is more complex than in the thin disk.

The trend of the data plotted in the left panel of Fig. 11 becomes even clearer in the $[\mathrm{La} / \mathrm{Y}]$ vs. $[\mathrm{La} / \mathrm{Fe}]$ plane (middle panel). Galactic stars and Sagittarius RGs display a smaller spread in $[\mathrm{La} / \mathrm{Y}]$ abundances at fixed $[\mathrm{La} / \mathrm{Fe}]$ abundance. Moreover, the separation between Sagittarius and Galactic stars becomes even more solid. Only the three most metal-poor objects in the Sagittarius sample overlap with Galactic thin disk stars. This finding indicates a strong correlation between $\mathrm{Y}$ and $\mathrm{Fe}$ over the entire metallicity range.
The right panel of Fig. 11 shows the same data, but they are plotted as a function of the iron abundance. The distribution in this plane is quite similar to the left panel, but with a larger spread in iron abundances. We note that the separation between Galactic and Sagittarius stars might be even more compelling than suggested by the current data. The 17 Cepheids with $[\mathrm{La} / \mathrm{Y}]>0.3$ come from the LIII sample. The authors did not take the hyperfine structure into account; moreover, 15 out of the 17 are located in the first quadrant and at Galactocentric distance larger than $9 \mathrm{kpc}$. Cepheids in the outer disk will play a crucial role in further constraining the use of the quoted chemical diagnostics to separate Galactic and dwarf galaxy stars.

In this context it is worth mentioning that the star Sgr 247 from the M13 sample lies off the main trend in the left and in the right panel of Fig. 11. The peculiar position of this object also shows up in the left and right panels of Fig. 10. The current findings further support the results by M13 suggesting that this object was polluted by more metal-poor $([\mathrm{Fe} / \mathrm{H}]$ ranging from about -0.5 to about -1.0 dex) AGB ejecta. The AGB yields in this object were polluted, but less than similar Sagittarius stars. This hypothesis is further supported by the evidence that the same object follows the main trend in the [hs/ls] and in the [s/r] abundance ratios (middle panels of Figs. 10 and 11).

\subsection{Comparison between predicted and observed [hs/ls] $s$-process index}

To further constrain the difference between the $s$-process index [hs/ls] in Cepheids with field Galactic stars and in nearby dwarf galaxies, Fig. 12 shows the comparison between theoretical and observed $[\mathrm{La} / \mathrm{Y}]$ as a function of iron content. The black lines display predicted final surface abundances for four low-mass (see labeled values) AGB models available on the FRUITY database ${ }^{2}$ (Cristallo et al. 2011, 2015b). The symbols and error bars for the data are the same as in the right panel of Fig. 11. The comparison brings forward several interesting new findings:

i) The agreement between theory and observations is quite good over the entire metallicity range covered by Cepheids. In this context it is worth mentioning that Cepheids offer a new opportunity to validate the [hs/ls] $s$-process index. Theoretical predictions are validated using a broad range

2 fruity.oa-teramo.inaf.it 
R. da Silva et al.: Neutron-capture elements across the Galactic thin disk using Cepheids
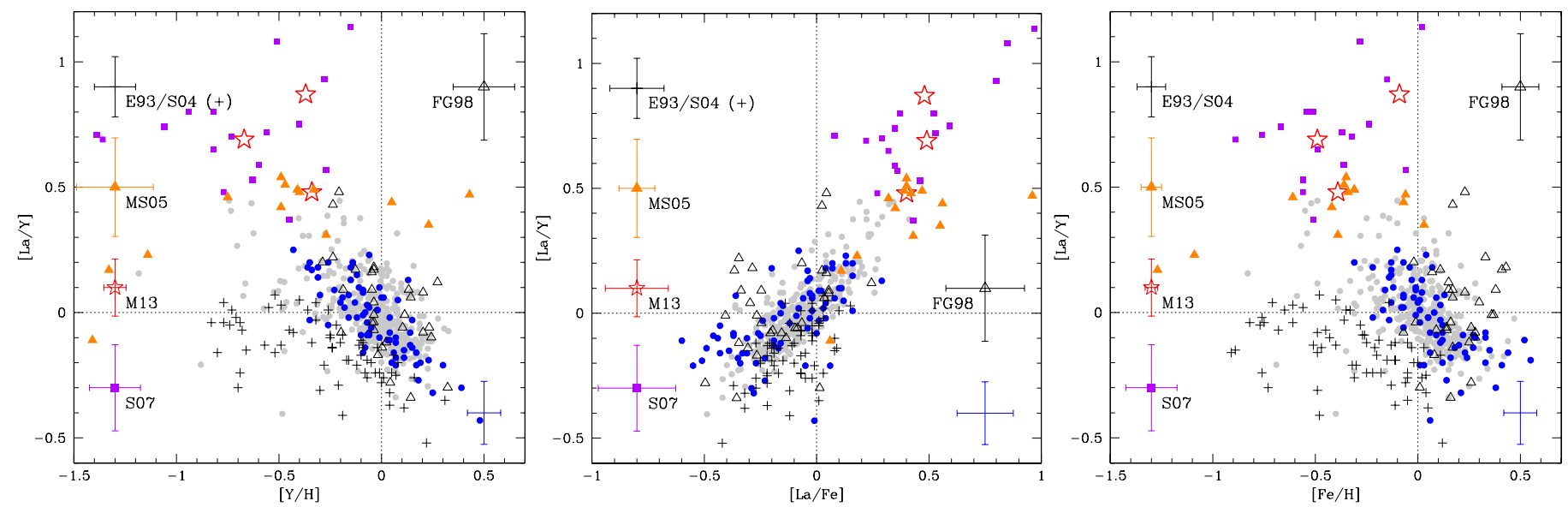

Fig. 11. Same as in Fig. 9, but for La and Y ratios. Sgr stars studied by Sbordone et al. (2007, S07, violet squares) are also plotted.

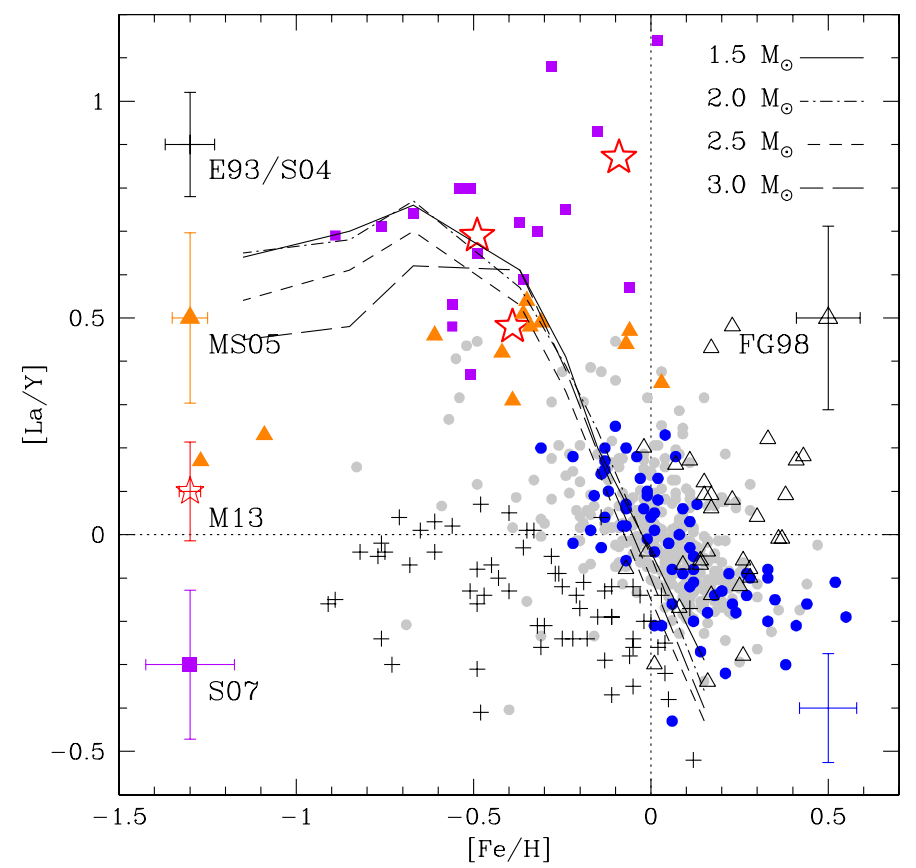

Fig. 12. Same as in the right panel of Fig. 11, but comparing the observational data with theoretical models available on the FRUITY database (Cristallo et al. 2011, 2015b).

of s-enhanced stars - O-rich and C-rich AGB stars, postAGB stars, Ba-rich stars, and $\mathrm{CH}$-rich stars - for which the evolutionary status is not well established. The advantage of using Cepheids is that they belong to the first stellar generation formed after the recent enrichment of the interstellar medium. The current comparison between theory and observations should be cautiously treated because we have not taken into account dilution effects and detailed chemical evolution models.

However, it is worth mentioning that the above comparison was performed overplotting predicted abundances on the observed values. This means that once corrected for the adopted solar abundances, we did not apply any shift to the predicted abundances. Data plotted in this figure suggest that predicted $[\mathrm{La} / \mathrm{Y}]$ abundances display a spread that is systematically smaller than observed in the metal-rich regime. The reader interested in a detailed discussion concerning the theoretical parameters affecting the spread of the above $s$-process index is referred to Cristallo et al. (2015a; see also Piersanti et al. 2013). In this context we would like to emphasize the similarity in the slope when moving from the metal-rich to the metal-poor regime of Galactic Cepheids. The current empirical uncertainties do not allow us to determine whether field dwarf stars provided by E93 and S04 show a shallower slope than Cepheids.

ii) Theory and observation display a steady increase in $[\mathrm{La} / \mathrm{Y}]$ when moving from the metal-rich into the metal-intermediate regime, i.e., $[\mathrm{Fe} / \mathrm{H}] \sim-0.4 /-0.7$. The $[\mathrm{La} / \mathrm{Y}]$ abundances, as expected, decrease in the metal-poor regime (Cristallo et al. 2009). There is a group of Sagittarius stars showing $[\mathrm{La} / \mathrm{Y}]$ abundances larger (i.e., $s$-process enhanced) than predicted by AGB models; these stars have already been discussed by M13. A similar discrepancy has also been found in CEMP stars at very low metallicities, which attain values of $s$-process index on the order of +1.3 dex (Spite \& Spite 2014; Beers et al. 2005). The lack of a sizable sample of Cepheids in the metal-intermediate regime do not allow us to provide independent constraints on the possible mismatch between predicted and observed [hs/ls] abundance ratios. We note that Mishenina et al. (2015), in a recent investigation of more than two dozen giant stars in five Galactic open clusters, found solid evidence of $[\mathrm{Ba} / \mathrm{Fe}]$ and $[\mathrm{Ba} / \mathrm{La}]$ enhancement. They suggested that the quoted empirical evidence might be explained by assuming a significant contribution from nonstandard $s$-process, i.e., the intermediate neutron-capture process suggested by Cowan \& Rose (1977).

To further validate the plausibility of the adopted theoretical framework for the production of $s$-process elements from AGB stars, we performed a plain test to constrain the slope of $[\mathrm{Y} / \mathrm{H}]$ vs. the Galactocentric distance. We performed a linear fit of the Cepheids plotted in Fig. 12 (i.e., [La/Y] vs. [Fe/H]). To overcome the increase in the spread in the more metal-poor and in the more metal-rich regimes we selected the objects with iron abundances between -0.3 and +0.3 dex. The current fit was combined with the analytical fits for $[\mathrm{Fe} / \mathrm{H}]$ and $[\mathrm{La} / \mathrm{H}]$ as a function of Galactocentric distance. We found that the expected slope for $[\mathrm{Y} / \mathrm{H}]$ as a function of Galactocentric distance is very similar to the observed slope ( -0.052 vs. $-0.053 \mathrm{dex} \mathrm{kpc}^{-1}$, respectively). This evidence indicates that $s$-process elements predicted by AGB models take into account the observed slopes among the investigated elements. 


\section{Summary and final remarks}

This is the tenth of a series of papers that focus on the metallicity distribution of the Galactic thin disk using classical Cepheids as stellar tracers. The DIsk Optical Near-infrared Young Stellar Object Spectroscopy (DIONYSOS) project provides homogeneous and accurate elemental abundances and distances for a significant fraction of the known Galactic Cepheids.

In this investigation we present accurate and homogeneous measurements of five neutron capture elements (Y, La, Ce, Nd, $\mathrm{Eu})$ for 73 Galactic classical Cepheids. The current abundances are based on high spectral resolution $(R \sim 38000)$ and high signal-to-noise ratio $(S / N \sim 50-300)$ spectra collected with UVES at ESO VLT. They were derived by accounting for the HFS of some lines of Y, La, and Eu, for which atomic data are available in the literature. The iron, $\alpha$ plus $\mathrm{Na}$ and $\mathrm{Al}$ abundances of the same Cepheids have already been discussed in Genovali et al. (2013, 2014, 2015). Our Cepheids are typical of the Galactic sample, and they cover a broad range of pulsation periods $(0.36 \leq \log P \leq \sim 1.54)$ and Galactocentric distances $\left(4.6 \leq R_{\mathrm{G}} \leq 14.3 \mathrm{kpc}\right)$.

We also selected similar abundances for Galactic Cepheids available in the literature and we ended up with homogenous measurements for 435 Galactic Cepheids. Roughly one-third of the entire sample has measurements provided by our group (current plus LEM), while the others come from LII, LIII, and YON. The different samples have from one dozen to four dozen Cepheids in common, which allowed us to provide homogeneous abundance scales for the quoted five elements plus iron and $\alpha$-elements (G14, G15).

The individual distances for the entire Cepheid sample are based on homogeneous NIR photometry, transformed into the 2MASS photometric system, and on the Period-Wesenheit relations provided by Inno et al. (2013). The main findings of the current analysis are the following:

i) $[$ element/H] radial gradients: the investigated neutron capture elements display well-defined radial gradients. The slopes for four ( $\mathrm{La}, \mathrm{Ce}, \mathrm{Nd}, \mathrm{Eu})$ of the five elements are average $\left(-0.025 \pm 0.004 \mathrm{dex} \mathrm{kpc}^{-1}\right)$. The $\mathrm{Y}$ slope is more than a factor of two steeper and more similar to the slopes of iron and $\alpha$-elements. The current estimates agree quite well with similar radial gradients available in the literature. However, we provide firm constraints concerning the $\mathrm{Nd}$ gradient for which a flat distribution was suggested when moving from the inner to the outer disk. Moreover, the difference in the slope between $\mathrm{Y}$ and the other three $s$-process elements $(\mathrm{La}, \mathrm{Ce}, \mathrm{Nd}$ ) brings forward a more complex enrichment history for this element.

ii) Comparison with theory: the comparison with radial gradients predicted by chemical evolution models provided by Cescutti et al. $(2006,2007)$ indicates a very good agreement for the slopes of both La and Eu.

iii) Comparison with observations: the comparison with similar abundances for field thin and thick dwarf and giant stars provided by E93, FG98, R03, S04, B05, and O13 indicates a very good agreement over the Galactocentric distances covered by the quoted samples.

iv) Age dependence: we took advantage of the strong anticorrelation between pulsation period and age to constrain the age dependence of the investigated elements. We found that the slopes are positive, i.e., they are more abundant in young (a few tens of Myrs) than in old ( $300 \mathrm{Myr})$ Cepheids. However, the slopes of $\mathrm{La}, \mathrm{Ce}$, and $\mathrm{Nd}$ are shallower than for iron, $\alpha$-elements, and light elements, while for $\mathrm{Y}$ and Eu they are more similar.

v) [element/Fe] radial gradients: we found that three $s$-process dominated elements ( $\mathrm{La}, \mathrm{Ce}, \mathrm{Nd}$ ) and one $r$-process dominated element $(\mathrm{Eu})$ display slopes that are on average a factor of two larger than similar slopes of the $\alpha$ - and light elements investigated by G15. The slope of $\mathrm{Y}$ is once again an exception, and indeed this element shows a flat distribution across the entire disk. The quoted trends are the consequence of the difference/similarity with the iron radial gradient.

vi) [element/Fe] abundance ratios in the super-metal-richregime: we found that $s$ - and $r$-process abundance ratios display a steady decrease for iron abundances larger than solar. The change in the slope indicates a clear contribution from $\mathrm{SNe}$ type Ia ejecta. The trend in the $[\mathrm{Eu} / \mathrm{Fe}]$ abundance ratio as a function of iron abundance further supports the above hypothesis with a steady decrease in the slope when moving from $[\mathrm{Fe} / \mathrm{H}] \sim-1.2$ to $[\mathrm{Fe} / \mathrm{H}] \sim-0.5$. The current findings support previous results for super metalrich field dwarfs by FG98.

vii) Spatial and temporal homogeneity: the reduced scatter in the above radial gradients at fixed Galactocentric distance and the lack of well-defined slopes for [element $/ \mathrm{Fe}$ ] as a function of the pulsation period (except for Ce) indicates that the chemical enrichment across the Galactic thin disk is characterized by firm spatial and temporal homogeneity.

viii) s-to-r abundance ratio: we found that Cepheid [La/Eu] abundance ratios show a well-defined anticorrelation when plotted as a function of Eu and Fe abundances. Field stars display a different trend. Indeed, they attain an almost constant ratio in the metal-poor regime and only for $[\mathrm{Eu} / \mathrm{H}]$ and $[\mathrm{Fe} / \mathrm{H}]$ larger than $\sim-0.5$ dex show a mild enhancement in La. The light s-to- $r$-process element abundance ratio ([Y/Eu]) shows a different trend. The Cepheids do not show a clear anticorrelation with $[\mathrm{Y} / \mathrm{H}]$ and with $[\mathrm{Y} / \mathrm{Fe}]$. On the other hand, field stars display a correlation with both $\mathrm{Y}$ and iron. Moreover, [Y/Eu] shows a well-defined correlation with iron abundance. This trend appears as the consequence of the strong correlation between $\mathrm{Y}$ and iron abundances.

ix) Heavy-to-light s element abundance ratio: we found that Cepheid $[\mathrm{La} / \mathrm{Y}]$ abundance ratios show a strong anticorrelation when plotted as a function of $\mathrm{Y}$ and $\mathrm{Fe}$ abundances. Field Galactic stars display the same trend, thus supporting the metallicity dependence of heavy (La, Ce, Nd) and light (Y) $s$-process elements on the metal content (Cristallo et al. 2009, 2011, 2015b). Moreover, we also found that the dispersion in $[\mathrm{La} / \mathrm{Y}]$ as a function of $[\mathrm{La} / \mathrm{Fe}]$ is small among Galactic and Sagittarius stars, further supporting similarity in the origin of $\mathrm{Fe}$ and $\mathrm{Y}$. Interestingly enough, we also found that in the quoted planes, in particular, in [La/Y] vs. $[\mathrm{La} / \mathrm{Fe}]$, the Sagittarius RGs are well separated by Galactic stars owing to their La enhancement, thus suggesting that they can be adopted as solid diagnostics to identify relic stars of dwarf galaxies accreted by our galaxies.

x) Comparison between predicted and observed s-process index: we performed a detailed comparison between predicted and observed $s$-process index $[\mathrm{La} / \mathrm{Y}]$. We found that final surface abundances of low-mass $\left(1.5 \leq M / M_{\odot} \leq 3.0\right)$ AGB stars agree quite well over the entire metallicity range covered by the current sample of classical Cepheids. 
Acknowledgements. This work was partially supported by PRIN-MIUR (2010LY5N2T) "Chemical and dynamical evolution of the Milky Way and Local Group galaxies" (P.I.: F. Matteucci) and PRIN-MIUR (20128PCN59) "Nucleosynthesis in AGB stars: an integrated approach" project (P.I.: L. Gialanella). We also acknowledge an anonymous referee for the positive opinions concerning this experiment and for the very pertinent suggestions that improved the content and the readability of the paper.

\section{References}

Anderson, R. I., Ekström, S., Georgy, C., et al. 2015, IAU Symp., 307, 206 Andrievsky, S. M., Kovtyukh, V. V., Luck, R. E., et al. 2002a, A\&A, 381, 32 Andrievsky, S. M., Bersier, D., Kovtyukh, V. V., et al. 2002b, A\&A, 384, 140 Andrievsky, S. M., Kovtyukh, V. V., Luck, R. E., et al. 2002c, A\&A, 392, 491 Andrievsky, S. M., Luck, R. E., Martin, P., \& Lépine, J. R. D. 2004, A\&A, 413, 159

Andrievsky, S. M., Lépine, J. R. D, \& Korotin, S. A. 2013, MNRAS, 428, 3252 Andrievsky, S. M., Luck, R. E., \& Korotin, S. A. 2014, MNRAS, 437, 2106

Ballester, P., Bramich, D., Forchi, V., et al. 2011, Astronomical Data Analysis Software and Systems XX, 442, 261

Beers, T. C., \& Christlieb, N. 2005, A\&ARv, 100, 32

Beers, T. C., Flynn, K., \& Gebhardt, K. 1990, AJ, 43, 531

Bensby, T., Feltzing, S., \& Lundström, I. 2003, A\&A, 410, 527 (B05)

Berger, E., Fong, W., \& Chornock, R. 2013, ApJ, 774, L23

Bisterzo, S., Gallino, R., Straniero, O., Cristallo, S., \& Käppeler, F. 2011, MNRAS, 418, 284

Bono, G. 2003, ASP Conf. Ser., 291, 45

Bono, G., Marconi, M., Cassini, S., et al. 2005, ApJ, 621, 966

Bono, G., Caputo, F., Marconi, M., \& Musella, I. 2010, ApJ, 715, 277

Bono, G., Genovali, K., Lemasle, B., et al. 2015, ASP Conf. Ser., 491, 148

Boyd, R. N., Famiano, M. A., Meyer, B. S., et al. 2012, ApJ, 744, 14

Burris, D. L., Pilachowski, C. A., Armandroff, T. E., et al. 2000, ApJ, 544, 302

Busso, M., Gallino, R., \& Wasserburg, G. J. 1999, ARA\&A, 37, 239

Busso, M., Gallino, R., Lambert., D. L., Travaglio, C., \& Smith, V. V. 2001, ApJ, 557,802

Cescutti, G., François, P., Matteucci, F., Cayrel, R., \& Spite, M. 2006, A\&A, 448,557

Cescutti, G., Matteucci, F., François, P., \& Chiappini, C. 2007, A\&A, 462, 943

Conti, P. S., \& Wallerstein, G. 1969, ApJ, 155, 11

Cowan, J. J., \& Rose, W. K. 1977, ApJ, 212, 149

Cristallo, S., Straniero, O., Gallino, R., et al. 2009, ApJ, 696, 797

Cristallo, S., Piersanti, L., Straniero, O., et al. 2011, ApJS, 197, 17

Cristallo, S., Abia, C., Straniero, O., \& Piersanti, L. 2015a, ApJ, 801, 53

Cristallo, S., Straniero, O., Piersanti, L., \& Gobrecht, D. 2015b, ApJS, 219, 40

Dékány, I., Minniti, D., Hajdu, G., et al. 2015, ApJ, 799, 11

Den Hartog, E. A., Lawler, J. E., Sneden, C., \& Cowan, J. J. 2003, ApJS, 148, 543

Edvardsson, B., Andersen, J., Gustafsson, B., et al. 1993, A\&A, 275, 101 (E93)

Feast, M. W., Menzies, J. W., Matsunaga, N., \& Whitelock, P. A. 2014, Nature, 509,342

Feltzing, S., \& Gustafsson, B. 1998, A\&AS, 129, 237 (FG98)

Gallino, R., Arlandini, C., Busso, M., et al. 1998, ApJ, 497, 388

Geisler, D., Smith, V. V., Wallerstein, G., Gonzalez, G., \& Charbonnel, C. 2005,

AJ, 129, 1428

Genovali, K., Lemasle, B., Bono, G., et al. 2013, A\&A, 554, A132

Genovali, K., Lemasle, B., Bono, G., et al. 2014, A\&A, 566, A37 (G14)

Genovali, K., Lemasle, B., da Silva, R., et al. 2015, A\&A, 580, A17 (G15)

Grevesse, N., Noels, A., \& Sauval, A. J. 1996, Cosmic Abundances, 99, 117

Grevesse, N., Scott, P., Asplund, M., \& Sauval, J. 2015, A\&A, 573, A27

Groenewegen, M. A. T., Udalski, A., \& Bono, G. 2008, A\&A, 481, 441

Gustafsson, B., Edvardsson, B., Eriksson, K., et al. 2008, A\&A, 486, 951

Harris, H. C. 1981, AJ, 86, 707

Harris, H. C., \& Pilachowski, C. A. 1984, ApJ, 282, 655

Inno, L., Matsunaga, N., \& Bono, G. 2013, ApJ, 764, 84

Kippenhahn, R., \& Smith, L. 1969, A\&A, 1, 142

Kovtyukh, V. V. 2007, MNRAS, 378, 617
Kovtyukh, V. V., \& Gorlova, N. I. 2000, A\&A, 358, 587

Kovtyukh, V. V., Wallerstein, G., \& Andrievsky, S. M. 2005, PASP, 117, 1182

Kraft, R. P. 1965, Galactic structure, eds. A. Blaauw, \& M. Schmidt (Chicago,

USA: University of Chicago Press), 157

Kraft, R. P., \& Schmidt, M. 1963, ApJ, 137, 249

Lawler, J. E., Bonvallet, G., \& Sneden, C. 2001a, ApJ, 556, 452

Lawler, J. E., Wickliffe, M. E., Den Hartog, E. A., \& Sneden, C. 2001b, ApJ, 563,1075

Leavitt, H. S. 1908, Annals of Harvard College Observatory, 60, 87

Leavitt, H. S., \& Pickering, E. C. 1912, Harvard College Observatory Circular, 173,1

Lemasle, B., François, P., Bono, G., et al. 2007, A\&A, 467, 283

Lemasle, B., François, P., Piersimoni, A., et al. 2008, A\&A, 490, 613

Lemasle, B., François, P., Genovali, K., et al. 2013, A\&A, 558, A31 (LEM)

Lemasle, B., de Boer, T. J. L., Hill, V., et al. 2014, A\&A, 572, A88

Lemasle, B., Kovtyukh, V. V., Bono, G., et al. 2015, A\&A, 579, A47

Letarte, B., Hill, V., Tolstoy, E., et al. 2010, A\&A, 523, A17

Luck, R. E. 2014, AJ, 147, 137

Luck, R. E., \& Lambert, D. L. 2011, AJ, 142, 136 (LIII)

Luck, R. E., Kovytuk, V. V., \& Andrievsky, S. M. 2006, AJ, 132, 902

Luck, R. E., Andrievsky, S. M., Kovtyukh, V. V., Gieren, W., \& Graczyk, D. 2011, AJ, 142, 51 (LII)

Lyubimkov, L. S., Lambert, D. L., Rostopchin, S. I., Rachkovskaya, T. M., \& Poklad, D. B. 2010, MNRAS, 402, 1369

Matsunaga, N., Kawadu, T., Nishiyama, S., et al. 2011, Nature, 477, 188

Matsunaga, N., Feast, M. W., Kawadu, T., et al. 2013, MNRAS, 429, 385

Martin, R. P., Andrievsky, S. M., Kovtyukh, V. V., et al. 2015, MNRAS, 449, 4071

McWilliam, A., \& Smecker-Hane, T. A. 2005, ASP Conf. Ser. 336, 221 (MS05)

McWilliam, A., Wallerstein, G., \& Mottini, M. 2013, ApJ, 778, 149 (M13)

Metzger, M. R., Caldwell, J. A. R., \& Schechter, P. L. 1992, AJ, 103, 529

Meyer-Hofmeister, E. 1969, A\&A, 2, 143

Minniti, D., Lucas, P. W., Emerson, J. P., et al. 2010, NewA, 15, 433

Mishenina, T., Pignatari, M., Carraro, G., et al. 2015, MNRAS, 446, 3651

Miville-Deschênes, M.-A., \& Lagache, G. 2005, ApJS, 157, 302

Nishimura, N., Takiwaki, T., \& Thielemann, F.-K. 2015, ApJ, 810, 109

Origlia, L., Oliva, E., Maiolino, R., et al. 2013, A\&A, 560, A46 (O13)

Pedicelli, S., Lemasle, B., Groenewegen, M., et al. 2010, A\&A, 518, L11

Piersanti, L., Cristallo, S., \& Straniero, O. 2013, ApJ, 774, 98

Pignatari, M., Gallino, R., Heil, M., et al. 2010, ApJ, 710, 1557

Pompéia, L., Hill, V., Spite, M., et al. 2008, A\&A, 480, 379

Pont, F., Kienzle, F., Gieren, W., \& Fouqué, P. 2001, A\&A, 376, 892

Raiteri, C. M., Gallino, R., Busso, M., Neuberger, D., \& Kaeppeler, F. 1993, ApJ, 419, 207

Reddy, B. E., Tomkin, J., Lambert, D. L., \& Prieto, C. A. 2003, MNRAS, 340, 304 (R03)

Sbordone, L., Bonifacio, P., Buonanno, R., et al. 2007, A\&A, 465, 815 (S07)

Scott, P., Asplund, M., Grevesse, N., Bergemann, M., \& Sauval, J. 2015, A\&A, 573, A26

Shetrone, M., Venn, K. A., Tolstoy, E., et al. 2003, AJ, 125, 684

Simmerer, J., Sneden, C., Cowan, J. J., et al. 2004, ApJ, 617, 1091 (S04)

Sneden, C., Cowan, J. J., \& Gallino, R. 2008, A\&ARv., 46, 241

Sousa, S. G., Santos, N. C., Israelian, G., Mayor, M., \& Monteiro, J. P. F. G. 2007, A\&A, 469, 783

Spite, M. 1967, Annales d'Astrophysique, 30, 211

Spite, M., \& Spite, F. 2014, Astron. Nachr., 335, 65

Takeda, Y., Kang, D.-I., Han, I., Lee, B.-C., \& Kim, K.-M. 2013, MNRAS, 432, 769

Thielemann, F.-K., Arcones, A., Käppeli, R., et al. 2011, Prog. Part. Nucl. Phys., 66,346

Tsujimoto, T., \& Nishimura, N. 2015, ApJ, 811, 10

Udalski, A., Szymańsky, M. K., \& Szymańsky, G. 2015, Acta Astron., 65, 1

Wanajo, S. 2013, ApJ, 770, 22

Wanajo, S., \& Janka, H.-T. 2012, ApJ, 746, 180

Windmark, F., Lindegren, L., \& Hobbs, D. 2011, A\&A, 530, A76

Woosley, S. E., \& Heger, A. 2015, ApJ, 810, 34

Yong, D., Carney, B. W., Teixera de Almeida, M. L., \& Pohl, B. L. 2006, AJ, $131,2256(\mathrm{YON})$ 